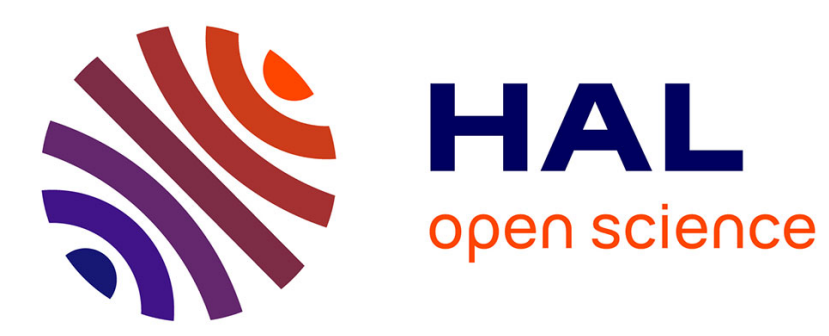

\title{
Convolution of orbital measures on symmetric spaces: a survey \\ P Graczyk, P Sawyer
}

\section{To cite this version:}

P Graczyk, P Sawyer. Convolution of orbital measures on symmetric spaces: a survey. 2014. hal$01085251 \mathrm{v} 2$

\section{HAL Id: hal-01085251 \\ https://hal.science/hal-01085251v2}

Preprint submitted on 25 Nov 2014

HAL is a multi-disciplinary open access archive for the deposit and dissemination of scientific research documents, whether they are published or not. The documents may come from teaching and research institutions in France or abroad, or from public or private research centers.
L'archive ouverte pluridisciplinaire HAL, est destinée au dépôt et à la diffusion de documents scientifiques de niveau recherche, publiés ou non, émanant des établissements d'enseignement et de recherche français ou étrangers, des laboratoires publics ou privés. 


\title{
Convolution of orbital measures on symmetric spaces: a survey
}

\author{
P. Graczyk and P. Sawyer \\ This paper is dedicated to Philip Feinsilver, Salah Mohammed, and Arunava Mukherjea at the \\ occasion of their retirement.
}

\begin{abstract}
This survey summarizes a long and fruitful collaboration of the authors on the properties of the convolutions of orbital measures on symmetric spaces or, equivalently, on the product formula for spherical functions on such spaces. A special accent is put on recent results in the singular case. They are presented in a simplified and unified manner in this survey.

New results for the flat symmetric spaces are also included, including a new short proof of the Thompson's conjecture in the complex case. Important open problems and possible applications of our results are proposed.
\end{abstract}

\section{Introduction}

1.1. Introductory example: uniform measures on spheres in $\mathbf{R}^{n}$. We start by discussing a simple and well known but fundamental example: the convolution of the uniform measures on centered spheres $S(r)$ in $\mathbf{R}^{n}$. Let $\delta_{S(r)}$ and $\delta_{S(R)}$ be two such measures. If $r \leq R$, the support $S(r)+S(R)$ of the convolution $\delta_{S(r)} * \delta_{S(R)}$ is equal to the annulus (see Figure 1)

$$
\left\{z \in \mathbf{R}^{n}: R-r \leq\|z\| \leq R+r\right\} .
$$

(we use the usual Euclidean norm on $\mathbf{R}^{n}$ ).

In order to prove the absolute continuity of $\delta_{S(r)} * \delta_{S(R)}$ when $r, R>0$, one uses classically a Fourier-based approach. The knowledge of the Fourier transform

$$
\mathcal{F}\left(\delta_{S(1)}\right)(y)=\Gamma\left(\frac{n}{2}\right)\left(\frac{2}{\|y\|}\right)^{n / 2-1} J_{n / 2-1}(\|y\|)
$$

where $J_{n / 2-1}$ is a Bessel function of the first kind (see e.g. [19, (2.30)]) allows one to study the inverse Fourier transform of $\mathcal{F}\left(\delta_{S(r)} * \delta_{S(R)}\right)$. This approach also leads to estimates and regularity properties of the density of $\delta_{S(r)} * \delta_{S(R)}$.

Let us look at this example from the point of view of the action of the compact group $K=\mathbf{O}(n)$ on the vector space $\mathbf{R}^{n}$.

1991 Mathematics Subject Classification. Primary 54C40, 14E20; Secondary 46E25, 20C20.

Key words and phrases. orbital measures, spherical functions, product formula, root systems, symmetric spaces, noncompact type. 


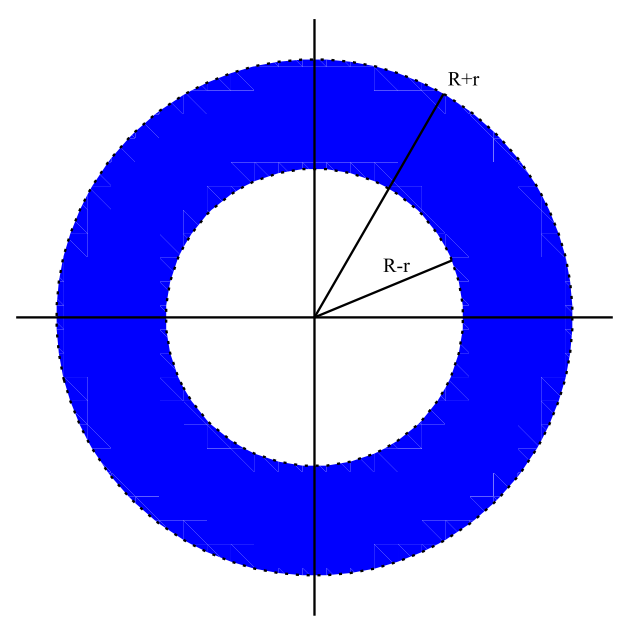

FiguRE 1. Support $S(r)+S(R)$

If $X \in \mathbf{R}^{n}$ and $X \neq 0$ then the orbit $K \cdot X$ is the sphere $S(\|X\|)$ of center 0 and radius $\|X\|$. The orbital measure $\delta_{X}^{\natural}$ on $K \cdot X$ is the transport of the Haar measure $m_{K}$ on $K$ by the map $k \in K \mapsto k \cdot X \in K \cdot X$. In other words, for a test function $f$ on $\mathbf{R}^{n}$

$$
\delta_{X}^{\natural}(f)=\int_{K} f(k \cdot X) d k
$$

We have $\delta_{X}^{\natural}=\delta_{S(\|X\|)}$.

Suppose now $X, Y \neq 0$ and then consider the convolution measure

$$
m_{X, Y}=\delta_{X}^{\natural} \star \delta_{Y}^{\natural}
$$

In other words, for a test function $f$ on $\mathbf{R}^{n}$

$$
m_{X, Y}(f)=\int_{\mathbf{O}(n) \times \mathbf{O}(n)} f\left(k_{1} X+k_{2} Y\right) d k_{1} d k_{2} .
$$

The measure $m_{X, Y}$ is the transport of the Haar measure $m_{K \times K}$ on $K \times K$ by the analytic map

$$
T\left(k_{1}, k_{2}\right)=k_{1} \cdot X+k_{2} \cdot Y .
$$

Let us now describe the support of $m_{X, Y}$, namely

$$
S_{X, Y}=\mathbf{O}(n) \cdot X+\mathbf{O}(n) \cdot Y
$$

which is the image of the group $\mathbf{O}(n) \times \mathbf{O}(n)$ by the map $T$. It is not difficult to see that

$$
S_{X, Y}=\|X\| \mathbf{O}(n) \cdot \mathbf{e}_{1}+\|Y\| \mathbf{O}(n) \cdot \mathbf{e}_{1}
$$

where $\mathbf{e}_{1}=(1,0, \ldots, 0)$. Without loss of generality, we can assume that $\|X\| \geq\|Y\|$. Observe that the set described in (1.2) is the annulus

$$
S_{X, Y}=\left\{Z \in \mathbf{R}^{n}:\|X\|-\|Y\| \leq\|Z\| \leq\|X\|+\|Y\|\right\} .
$$


Indeed, we have $\left\|S_{X, Y}\right\|=[\|X\|-\|Y\|,\|X\|+\|Y\|]$ which means that for every $r$ in this interval, there is a $Z \in S_{X, Y}$ with $\|Z\|=r$. Given that $S_{X, Y}$ is invariant under the action of $\mathbf{O}(n)$, the rest follows.

In order to study the existence of the density of $m_{X, Y}$, some basic differential geometry arguments explained in the beginning of the Section 2 (see Theorem 2.3) justify the equivalence of the following properties

- The measure $m_{X, Y}=T\left(m_{K \times K}\right)$ is absolutely continuous

- The support $S_{X, Y}=T(K \times K)$ has a nonempty interior

- The derivative of the map $T$ is surjective at a point $\left(k_{1}, k_{2}\right)$.

This gives two new approaches to the question of absolute continuity of $m_{X, Y}$ : the support approach and the surjectivity approach.

Let us illustrate these two other methods on the introductory example.

The annulus $S_{X, Y}$ has nonempty interior if and only if $X$ and $Y \neq 0$. Thus, by the support approach, the measure $m_{X, Y}=T\left(m_{K \times K}\right)$ is absolutely continuous if and only if $X$ and $Y \neq 0$.

The surjectivity approach is also very simple. The image of the derivative of the map $T$ at $\left(k_{1}, k_{2}\right)$ is easily seen to be $k_{1} \mathfrak{s o}(n) X+k_{2} \mathfrak{s o}(n) Y$. There exists $\left(k_{1}, k_{2}\right)$ such that $d T$ is surjective if and only if $k_{1} \mathfrak{s o}(n) X+k_{2} \mathfrak{s o}(n) Y=\mathbf{R}^{n}$ or if $\mathfrak{s o}(n) X+k \mathfrak{s o}(n) Y=\mathbf{R}^{n}$ (taking $k=k_{1}^{-1} k_{2}$ ) for some $k$. If, as before, we assume that $X=\|X\| \mathbf{e}_{1}$ and $Y=\|Y\| \mathbf{e}_{1}$, the condition becomes $\left[0, a_{2}, \ldots, a_{n}\right]^{T}+$ $k\left[0, b_{2}, \ldots, b_{n}\right]^{T}=\mathbf{R}^{n}$ for some $k$ (the $a_{i}$ 's and $b_{i}$ 's being arbitrary). The last condition is easily satisfied.

The main objective of our study of convolutions of $K$-orbital measures $\delta_{g}^{\natural}$ on Riemannian symmetric spaces $G / K$ is the existence of their density. Even though the spherical Fourier transform of such measures is known to be the spherical function $\phi_{\lambda}(g)$, the Fourier-based approach does not lead to sharp conditions due to the lack of efficient estimates of $\phi_{\lambda}(g)$ for singular $g$ 's.

However, the support approach and the surjectivity approach can be used. That's why we wanted to explain them on the introductory example, where they are particularly simple and rather unknown, in favor of the Fourier-based approach.

Let us notice that our introductory example can be realized as the Euclidean symmetric space $\mathbf{O}(n) \rtimes \mathbf{R}^{n} / \mathbf{O}(n)$ as discussed in Section 4 .

1.2. Basic notations. Let $G / K$ be a Riemannian symmetric space of noncompact type (Helgason provides an excellent introduction in [16]):

Here $G$ is a semisimple Lie group of noncompact type with finite centre and $K$ is a maximal compact subgroup of $G$. Let $\mathfrak{a}^{+}$be the positive Weyl chamber. The Cartan decomposition of the group $G$ writes

$$
g=k_{1} e^{a(g)} k_{2}
$$

where $k_{1}, k_{2} \in K$ and $a(g) \in \overline{\mathfrak{a}^{+}}$is uniquely determined.

We define now the main objects of our work, the orbital measures and their convolutions.

Consider $X, Y \in \mathfrak{a}$ and let $m_{K}$ denote the Haar measure of the group $K$. We define

$$
\delta_{e^{X}}^{\natural}=m_{K} \star \delta_{e^{x}} \star m_{K} \cdot
$$




\begin{tabular}{|l|c|c|}
\hline Measures & Lives on & support \\
\hline$\delta_{e^{X}}^{\natural}=m_{K} \star \delta_{e^{X} \star m_{K}}$ & $G$ & $K e^{X} K$ \\
\hline$m_{X, Y}=\delta_{e^{X}}^{\natural} \delta_{e^{Y}}^{\natural}$ & $G$ & $K e^{X} K e^{Y} K$ \\
\hline $\begin{array}{c}\mu_{X, Y}=\text { Transport of } m_{X, Y} \\
\text { on } \overline{\mathfrak{a}^{+}} \text {by the map } \\
g \rightarrow a(g)\end{array}$ & $\overline{\mathfrak{a}^{+}}$ & $a\left(e^{X} K e^{Y}\right)$ \\
& & \\
\hline
\end{tabular}

TABLE 1. Important measures

1.3. Motivations and applications. The problem of the absolute continuity of the convolution of two orbital measures

$$
m_{X, Y}=\delta_{e^{X}}^{\natural} \star \delta_{e^{Y}}^{\natural}
$$

and the study of the properties of its density that we address in this survey have important applications in the harmonic analysis of spherical functions on $G / K$ and in probability theory.

1.3.1. Applications in harmonic analysis: product formula. The spherical Fourier transform of $\delta_{e^{X}}^{\natural}$ is equal to the spherical function $\phi_{\lambda}\left(e^{X}\right)$ where $\lambda$ is a complexvalued linear form on $\mathfrak{a}$.

Thus the product $\phi_{\lambda}\left(e^{X}\right) \phi_{\lambda}\left(e^{Y}\right)$ is the spherical Fourier transform of the convolution $m_{X, Y}=\delta_{e^{X}}^{\natural} \star \delta_{e^{Y}}^{\natural}$.

Therefore, using the fact that the probability measure $\mu_{X, Y}$ is the Cartantransport of $m_{X, Y}$ on $\overline{\mathfrak{a}^{+}}$,

$$
\phi_{\lambda}\left(e^{X}\right) \phi_{\lambda}\left(e^{Y}\right)=\int_{\overline{\mathfrak{a}}^{+}} \phi_{\lambda}\left(e^{H}\right) d \mu_{X, Y}(H)
$$

The last formula is a version of the product formula for spherical functions on $G / K$. Like Helgason $[\mathbf{1 6}, \mathbf{1 8}]$, we reserve the name of product formula to the case when $\mu_{X, Y}$ has a density $k(H, X, Y)$. Thus the product formula for spherical functions

$$
\phi_{\lambda}\left(e^{X}\right) \phi_{\lambda}\left(e^{Y}\right)=\int_{\mathfrak{a}^{+}} \phi_{\lambda}\left(e^{H}\right) k(H, X, Y) \delta(H) d H,
$$

where $\delta(H) d H$ is the Cartan-transport of the invariant measure on $G / K$, is equivalent to the absolute continuity of the measure $\mu_{X, Y}$. Given that $\phi_{\lambda}\left(e^{X}\right) \phi_{\lambda}\left(e^{Y}\right)=$ $\int_{K} \phi_{\lambda}\left(e^{X} k e^{Y}\right) d k$, formula (1.3) is equivalent to

$$
\int_{K} f\left(e^{X} k e^{Y}\right) d k=\int_{\mathfrak{a}^{+}} f\left(e^{H}\right) k(H, X, Y) \delta(H) d H
$$

for every $K$-biinvariant function $f$.

The investigation of the product formula was initiated by Helgason [16, Prop.IV.10.13, p.480]. Helgason proposes in [18, p. 367] studying the properties of $\mu_{X, Y}$ in relation to the structure of $G$ as an interesting open problem. The questions of existence and explicit expression of a density of $\mu_{X, Y}$ are thus of great importance. The investigation of this problem was started by Flensted-Jensen and Koornwinder on hyperbolic spaces $[\mathbf{4}, \mathbf{2 3}]$. These questions were investigated by Rösler and other authors (see $[\mathbf{3 0}]$ and references therein) with the hope of generalization in the Dunkl and hypergroups setting. 
1.3.2. Applications in probability. They concern two groups of problems:

(1) Arithmetic of probability measures. In order to characterize the important class $I_{0}$ of probability laws without indecomposable factors (in the sense of the convolution product), Ostrovskii [27], Trukhina [34] and Voit [35] use as the main tool the product formula and some properties of its kernel, respectively on $\mathbf{R}^{n} / \mathbf{O}(n)$, on real hyperbolic spaces and on some hypergroups. We conjecture that our results will allow the characterization of the $I_{0}$ class on all symmetric spaces $G / K$ as $K$-invariant Gaussian measures.

(2) Properties of random walks on semi-simple groups. The property of absolute continuity of sufficiently large convolution powers $\left(\delta_{e}^{\natural}\right)^{l}$ is essential in the study of random walks on groups (see for example $[\mathbf{2 0}, \mathbf{2 1}, \mathbf{2 4}]$ ). The measures, with a certain convolution power allowing an absolutely continuous part, are called spreadout. We also believe that the results on the convolution powers of $\delta_{e x}^{\natural}$ will be useful in the study of isotropic $K$-invariant random walks on $G / K$, see $[\mathbf{6}]$ in the case $\mathbf{R}^{n}$.

1.4. Plan of the survey. We present here the organization of this survey.

In Section 2 we present the results on the existence of the density of the measures $m_{X, Y}$ and $\mu_{X, Y}$.

In Section 3 we discuss formulas available for the density and we give a description of the support.

We conclude with Section 4 with a discussion of our problems in the case of Euclidean (or flat) symmetric spaces. This section, in particular, contains new results.

\section{Absolute continuity of the convolution of orbital measures}

Our goal in this section is to discuss sharp criteria guaranteeing the existence of the density of the convolution of two orbital measures. We will develop the results in a variety of symmetric spaces of noncompact type and show in Section 4 that they apply in the case of symmetric spaces of Euclidean type.

Here are the three main Problems that we are discussing in this survey:

(1) Prove the existence of the density when $X$ is regular and $Y \neq 0$.

(2) Provide a sufficient and necessary condition for the existence of the density when $X$ and $Y$ are both singular.

(3) Provide a sufficient and necessary condition for the existence of the density of $\delta_{e^{X_{1}}}^{\natural} \ldots * \delta_{e^{x_{m}}}^{\natural}$ and $\left(\delta_{e^{X}}^{\natural}\right)^{l}$.

2.1. Existence of the density: methods. We will discuss here the various tools and equivalences that we have developed to prove the existence of the density.

Here are the methods we used in our research in order to show the absolute continuity of measures $m_{X, Y}$ and $\mu_{X, Y}$.

(i) Support approach for Problem 1. Show that $\operatorname{supp}\left(\mu_{X, Y}\right)=a\left(e^{X} K e^{Y}\right)$ has a nonempty interior using local Taylor expansions of the map $t \rightarrow$ $a\left(e^{X} k_{t}^{X_{\alpha}} e^{Y}\right)$ at $X+Y($ see $[\mathbf{1 3}])$. Here $X_{\alpha}$ denotes a root vector and $k_{t}^{X_{\alpha}}=\exp \left(t\left(X_{\alpha}+\theta X_{\alpha}\right)\right)$.

(ii) Fourier-based approach for Problem 1. Using inverse spherical Fourier transform (see [11]). 
(iii) Surjectivity approach for Problems $\mathbf{2}$ and $\mathbf{3}$. This technique was introduced and used in $[\mathbf{7}, \mathbf{8}, \mathbf{9}]$ and is based on the study of the surjectivity of the differential of the map $T\left(k_{1}, k_{2}, k_{3}\right)=k_{1} e^{X} k_{2} e^{Y} k_{3}$, equivalent to Theorem 2.3, part (7).

From today's perspective, it is clear for us that the surjectivity approach (2.1) is the simplest and the most powerful tool allowing a unified treatment of all three Problems 1, 2, 3. That is why we present this method in detail in this survey. However, the lemma that follows which was proven in $[\mathbf{9}]$ will be used in conjunction with the "support approach" to prove that the criteria that we are proposing are necessary (i.e. sharp).

LEMma 2.1. Let $U=\operatorname{diag}\left([\overbrace{u_{0}, \ldots, u_{0}}^{r}, u_{1}, \ldots, u_{N-r}]\right.$ and $V=\operatorname{diag}\left([\overbrace{v_{0}, \ldots, v_{0}}^{s}, v_{1}, \ldots, v_{N-s}]\right.$ where $r+s>N, s<N, r<N$ and the $u_{i}$ 's and $v_{j}$ 's are arbitrary. Then each element of a $\left(e^{U} \mathbf{S U}(N, \mathbf{F}) e^{V}\right)$ has at least $r+s-N$ entries equal to $u_{0}+v_{0}$ (here $a(g)$ corresponds to the singular values of $g$ ).

REMARK 2.2. The following two observations are used repeatedly in our work on the product formula:

(1) Let $f: M \rightarrow \mathbf{R}$ (or $\mathbf{C}$ ) be a nonzero analytic function where $M$ is a real (or complex) analytic manifold. Then $U=\{m: f(m) \neq 0\}$ is a dense open set of $M$.

(2) The intersection of two dense open sets of $M$ is a dense open set of $M$.

Observation 2 is simple but useful: it will be used to show that two properties that are each valid almost everywhere are valid together almost everywhere.

Theorem 2.3. Let $F(k)=a\left(e^{X} k e^{Y}\right)$ and let $T: K \times K \times K \rightarrow G$ be defined by $T\left(k_{1}, k_{2}, k_{3}\right)=k_{1} e^{X} k_{2} e^{Y} k_{3}$. The following statements are equivalent:

(1) $\mu_{X, Y}$ is absolutely continuous.

(2) $\operatorname{supp}\left(\mu_{X, Y}\right)=a\left(e^{X} K e^{Y}\right)$ has a nonempty interior.

(3) There exists $k \in K$ such that $\left.d F\right|_{k}$ is surjective.

(4) $m_{X, Y}$ is absolutely continuous.

(5) $\operatorname{supp}\left(m_{X, Y}\right)=K e^{X} K e^{Y} K$ has a nonempty interior.

(6) There exists $\left(k_{1}, k_{2}, k_{3}\right) \in K \times K \times K$ such that $\left.d T\right|_{\left(k_{1}, k_{2}, k_{3}\right)}$ is surjective.

(7) Let $V_{X}=\operatorname{span}\left\{X_{\alpha}-\theta\left(X_{\alpha}\right): \alpha(X) \neq 0\right\} \subset \mathfrak{p}$ (similarly for $V_{Y}$ ).

$$
\exists k \in K \quad V_{X}+A d(k) V_{Y}=\mathfrak{p} .
$$

Proof. We first observe that $m_{X, Y}$ is absolutely continuous if and only if its support $K e^{X} K e^{Y} K$ has nonempty interior $U$. This implies that the derivative of $T$ is surjective at least for some $\mathbf{k}$. Indeed, by Sard's theorem (see for example [16, p. 479] and the reference therein), given that $T$ is analytic, on the set $C$ of its critical points where $d T$ is not surjective, the invariant measure of $T(C)$ is zero. Given that the image of $T$ contains an open set, $C$ cannot be the whole of $K \times K \times K$. Hence, $T$ is surjective at least at one point and therefore, using Remark 2.2, $d T$ is surjective for every $\left(k_{1}, k_{2}, k_{3}\right)$ in a dense open subset of $K \times K \times K$. Using the implicit function theorem, a density $k_{G}(\cdot, X, Y)$ exists for the measure $m_{X, Y}$. On the other hand, if the image of $T$ does not contain an open set, the set of critical points will be $C=K \times K \times K$ and the invariant measure of $T(K \times K \times K)$ is zero. Therefore, the measure $m_{X, Y}$ is not absolutely continuous. 
The same result is true for the measure $\mu_{X, Y}$ although the proof requires some adjustment since $F(k)=a\left(e^{X} k e^{Y}\right)$ is continuous but not necessarily analytic everywhere. In [13], we worked around this technical difficulty by observing that $\mathfrak{a}$ is analytic on $G^{\prime}=K e^{\mathfrak{a}^{++}} K$ where $\mathfrak{a}^{++}=\left\{H \in \mathfrak{a}^{+}: \alpha(H) \neq \beta(H)\right.$ for $\left.\alpha \neq \beta\right\}$.

Rather than reproducing here all the details, we can observe that $\mu_{X, Y}$ is absolutely continuous if and only if $m_{X, Y}$ is and that the interiors of their supports are closely related. If $k(H, X, Y)$ and $k_{G}(g, X, Y)$ are the respective densities of $\mu_{X, Y}$ and $m_{X, Y}$ then

$$
\begin{aligned}
k(H, X, Y) & =\int_{K \times K} k_{G}\left(k_{1} e^{H} k_{2}, X, Y\right) d k_{1} d k_{2}, \\
k_{G}(g, X, Y) & =k(a(g), X, Y) .
\end{aligned}
$$

Finally, we show that (6) allows us to deduce another more practical criterion. We have

$$
\begin{aligned}
d T_{\mathbf{k}}(A, B, C) & =\left.\frac{d}{d t}\right|_{t=0} e^{t A} k_{1} e^{X} e^{t B} k_{2} e^{Y} e^{t C} k_{3} \\
& =A k_{1} e^{X} k_{2} e^{Y} k_{3}+k_{1} e^{X} B k_{2} e^{Y} k_{3}+k_{1} e^{X} k_{2} e^{Y} C k_{3} \\
(2.2) \quad & =k_{1} e^{X}\left[\operatorname{Ad}\left(e^{-X}\right)\left(k_{1}^{-1} A k_{1}\right)+B+\operatorname{Ad}\left(k_{2}\right) \operatorname{Ad}\left(e^{Y}\right)\left(k_{3} C k_{3}^{-1}\right)\right] k_{2} e^{Y} k_{3} .
\end{aligned}
$$

We conclude from (2.2) that $d T_{\mathbf{k}}$ is surjective if and only if there exists $k \in K$ such that

$$
\begin{aligned}
\operatorname{Ad}\left(e^{-X}\right) \mathfrak{k}+\mathfrak{k}+\operatorname{Ad}(k) \operatorname{Ad}\left(e^{Y}\right) \mathfrak{k} & =\mathfrak{g} \text { or } \\
{\left[\mathfrak{k}+\operatorname{Ad}\left(e^{-X}\right) \mathfrak{k}\right]+\operatorname{Ad}(k)\left[\mathfrak{k}+\operatorname{Ad}\left(e^{Y}\right) \mathfrak{k}\right] } & =\mathfrak{g} .
\end{aligned}
$$

If we project this equation on $\mathfrak{p}$ via the map $X \rightarrow X-\theta(X)$, the criterion (6) becomes equivalent to the existence of $k \in K$ such that (2.1) holds. Indeed, given that

$$
\begin{gathered}
k+\operatorname{Ad}\left(e^{-X}\right) \mathfrak{k}=\operatorname{span}\left\{X_{\alpha}+\theta\left(X_{\alpha}\right), e^{\alpha(X)} X_{\alpha}+e^{-\alpha(X)} \theta\left(X_{\alpha}\right)\right\} \\
=\operatorname{span}\left\{X_{\alpha}+\theta\left(X_{\alpha}\right), \frac{e^{\alpha(X)}+e^{-\alpha(X)}}{2}\left(X_{\alpha}+\theta\left(X_{\alpha}\right)\right)\right. \\
\left.\quad+\frac{e^{\alpha(X)}-e^{-\alpha(X)}}{2}\left(X_{\alpha}-\theta\left(X_{\alpha}\right)\right)\right\}
\end{gathered}
$$

the result follows.

If we refer to Remark 2.2 , it is helpful to note that in (3) and (6), we could write instead "There exists a dense open subset $U$ of $K$ (or of $K \times K \times K$ ) such that $\left.d F\right|_{k}$ (or $\left.\left.d T\right|_{\left(k_{1}, k_{2}, k_{3}\right)}\right)$ is surjective everywhere on $U . "$

2.2. Existence of the density: symmetric spaces of noncompact type. We believe that the simplest method to prove the statement of Problem $\mathbf{1}$ is the surjectivity approach via Theorem 2.3 , part (7). Below, we provide a new proof of Theorem 2.4 using Theorem 2.3, part (7). In [13], we showed that:

TheOREm 2.4. Let $G / K$ be an irreducible symmetric space of noncompact type. If $X \in \mathfrak{a}$ is regular and $Y \in \mathfrak{a}$ is not 0 , then the convolution $\delta_{e^{X}}^{\natural} * \delta_{e^{Y}}^{\natural}$ is absolutely continuous. 
The proof that we used in [13] to show that $\left.d F\right|_{k}$ is surjective for some $k \in K$ was based on local Taylor expansions at $X+Y$. It goes like this: $a\left(e^{X} e^{t\left(X_{\alpha}+\theta\left(X_{\alpha}\right)\right)} e^{Y}\right)=$ $X+Y+S t^{2} H_{\alpha}+O\left(t^{2}\right)$ where $S \neq 0$ whenever $\alpha(X) \neq 0$ and $\alpha(Y) \neq 0$. If we get enough directions to generate $\mathfrak{a}$ (we get every $H_{\alpha}, \alpha>0$ when $X, Y \in \mathfrak{a}^{+}$) then the density has to exist. We will show here that the criterion given in (2.1) can be used to prove Theorem 2.4.

We reproduce here the following result from $[\mathbf{1 3}]$ :

LEMma 2.5. Let $\Delta$ be an irreducible root system and $\Delta_{0}$ be the set of simple positive roots. If $\beta_{1} \in \Delta_{0}$ then one may order the elements of $\Delta_{0}$ in such a way $\beta_{2}, \ldots, \beta_{r}\left(r=\left|\Delta_{0}\right|\right)$ that $\sum_{i=1}^{k} \beta_{i} \in \Delta$ for all $k=1, \ldots, r$.

Proof. We use induction on $r$. The result is trivial when $r=1$.

Suppose that the statement is true for any root system with $r-1$ simple positive roots. In this paper, the scalar product on $\mathfrak{a}$ (and correspondingly on $\mathfrak{a}^{*}$ ) is defined via the Killing form: for $X, Y \in \mathfrak{a},\langle X, Y\rangle=B(X, Y)$.

Consider the Dynkin diagram $D$ of a root system with $\left|\Delta_{0}\right|=r$. As a graph, this diagram is a finite tree so there exists a root $\alpha_{r} \in \Delta$ such that $\left\langle\alpha, \alpha_{r}\right\rangle \neq 0$ for only one other root in $\Delta$, say $\alpha=\alpha_{r-1}$ (geometrically, it means that the only vertex of $D$ connected with $\alpha_{r}$ is $\alpha_{r-1}$ ).

Let $\Delta^{\prime}$ be the root system generated by the simple roots $\Delta_{0} \backslash\left\{\alpha_{r}\right\}$ (the Dynkin diagram $D^{\prime}$ of $\Delta^{\prime}$ is obtained from $D$ by suppressing the vertex $\alpha_{r}$ and the edge $\left.\left[\alpha_{r-1}, \alpha_{r}\right]\right)$.

Let us order the elements of $\Delta_{0} \backslash\left\{\alpha_{r}\right\}$ in such a way $\alpha_{1}, \ldots, \alpha_{r-1}$ that $\sum_{i=k}^{r-1} \alpha_{i} \in \Delta$ for all $k=1, \ldots, r-1$. This is possible by the induction hypothesis.

Let $\beta=\alpha_{1}+\cdots+\alpha_{r-1} \in \Delta$. As $\left\langle\alpha_{p}, \alpha_{r}\right\rangle=0$ when $p<r-1$ and $\left\langle\alpha_{r-1}, \alpha_{r}\right\rangle<0$ (all this follows from the fact that $D$ contains the edge $\left[\alpha_{r-1}, \alpha_{r}\right]$ and no other edge with vertex $\alpha_{r}$ ) we infer that $\left\langle\beta, \alpha_{r}\right\rangle<0$. This implies [16, Lemma 2.18 page 291] that $\beta+\alpha_{r}=\alpha_{1}+\cdots+\alpha_{r} \in \Delta$. The statement of the lemma is then true for $\beta_{1}=\alpha_{i}, i<r$. It is also true for $\beta_{1}=\alpha_{r}$ since $\sum_{i=k}^{r-1} \alpha_{i}+\alpha_{r} \in \Delta$ for $k=1, \ldots$, $r-1$ which follows from the same argument replacing $\beta$ by $\alpha_{k}+\cdots+\alpha_{r-1}$.

The lemma could also be proven using the classification of root systems by a case by case examination (see for instance $[\mathbf{1 6}]$ ).

Proof (of the theorem). Let $r$ be the rank of the symmetric space. We can assume without loss of generality that $X \in \mathfrak{a}^{+}$and that $Y \in \overline{\mathfrak{a}^{+}}$. We will show that

$$
V_{X}+\operatorname{Ad}(k) V_{Y}=\mathfrak{p} .
$$

Since $X$ is regular, we have $\mathfrak{p}=V_{X} \oplus \mathfrak{a}$ and therefore, $\operatorname{dim} V_{X}=\operatorname{dim} \mathfrak{p}-r$. Since $Y \neq 0$, there exists a simple $\operatorname{root} \beta_{1}$ such that $\beta_{1}(Y)>0$. Let $\beta_{i}, i=2$, $\ldots, r$ be as in Lemma 2.5. Let $\gamma_{k}=\sum_{i=1}^{k} \beta_{i}$ and note that $\gamma(Y)>0$ for each $k$. If $H_{\gamma_{k}}=\left[X_{\gamma_{k}}, \theta\left(X_{\gamma_{k}}\right)\right]$ then the $H_{\gamma_{k}}$ 's are linearly independent and generate $\mathfrak{a}$. Indeed, $\sum_{k=1}^{r} b_{k} H_{\gamma_{k}}=0$ implies $\sum_{k=1}^{r} b_{k} B\left(H_{\gamma_{k}}, H\right)=0$ for all $H \in \mathfrak{a}$. We then have $\sum_{k=1}^{r} b_{k} \gamma_{k}(H)=0$ which is equivalent to $\sum_{k=1}^{r} \sum_{i=1}^{k} b_{k} \beta_{i}(H)=0$ for all $H \in \mathfrak{a}$. This means that $\sum_{k=1}^{r} \sum_{i=1}^{k} b_{k} \beta_{i}=0$ or $\sum_{i=1}^{r}\left(\sum_{k=i}^{r} b_{k}\right) \beta_{i}=0$ from which we easily conclude that all the $b_{i}$ 's must be 0 .

Let

$$
k_{t}=\exp \left(t \sum X_{\gamma_{j}}^{\mathfrak{k}}\right), \quad X_{\gamma_{j}}^{\mathfrak{k}}=X_{\gamma_{j}}+\theta X_{\gamma_{j}} .
$$


Then, writing $X^{\mathfrak{p}}=X-\theta X \in \mathfrak{p}$,

$$
\operatorname{Ad}\left(k_{t}\right)\left(X_{\gamma_{j}}^{\mathfrak{p}}\right)=f(t) H_{\gamma_{j}}
$$

modulo $V_{X}$ with $f(t)=-2 t+o(t)$ as $t$ tends to 0 .

Now, provided that $t$ is small enough so that the terms in $t H_{\gamma_{j}}$ do not disappear, we have:

$$
\begin{aligned}
V_{X} \oplus \operatorname{span}\left\{\operatorname{Ad}\left(k_{t}\right)\left(X_{\gamma_{1}}^{\mathfrak{p}}\right)\right\} & =V_{X} \oplus \operatorname{span}\left\{H_{\gamma_{1}}\right\} \\
V_{X} \oplus \operatorname{span}\left\{\operatorname{Ad}\left(k_{t}\right)\left(X_{\gamma_{1}}^{\mathfrak{p}}\right), \operatorname{Ad}\left(k_{t}\right)\left(X_{\gamma_{1}}^{\mathfrak{p}}\right)\right\} & =V_{X} \oplus \operatorname{span}\left\{H_{\gamma_{1}}, H_{\gamma_{2}}\right\} \\
\ldots & \\
V_{X} \oplus \operatorname{span}\left\{\operatorname{Ad}\left(k_{t}\right)\left(X_{\gamma_{1}}^{\mathfrak{p}}\right), \ldots, \operatorname{Ad}\left(k_{t}\right)\left(X_{\gamma_{r}}^{\mathfrak{p}}\right)\right\} & =V_{X} \oplus \operatorname{span}\left\{H_{\gamma_{1}}, \ldots, H_{\gamma_{r}}\right\} \\
& \subset V_{X}+\operatorname{Ad}(K) V_{Y} .
\end{aligned}
$$

Since $V_{X} \oplus \operatorname{span}\left\{H_{\gamma_{1}}, \ldots, H_{\gamma_{r}}\right\}=\mathfrak{p}$, we have proven the Theorem.

The density can however still exist when both $X$ and $Y$ are singular. In the case of the root system $A_{2}$, if $X$ and $Y$ are singular, then the density does not exist. Indeed, if $X=x \operatorname{diag}[1,1,-1], Y=y \operatorname{diag}[1,1,-1]$ and $k \in \mathbf{S O}(3)$ then

$$
\begin{aligned}
& a\left(e^{X} k e^{Y}\right) \\
& =a\left(e^{X}\left[\begin{array}{ccc}
\cos \theta_{1} & -\sin \theta_{1} & 0 \\
\sin \theta_{1} & \cos \theta_{1} & 0 \\
0 & 0 & 1
\end{array}\right]\left[\begin{array}{ccc}
1 & 0 & 0 \\
0 & \cos \theta_{2} & -\sin \theta_{2} \\
0 & \sin \theta_{2} & \cos \theta_{2}
\end{array}\right]\left[\begin{array}{ccc}
\cos \theta_{3} & -\sin \theta_{3} & 0 \\
\sin \theta_{3} & \cos \theta_{3} & 0 \\
0 & 0 & 1
\end{array}\right] e^{Y}\right) \\
& =a\left(\left[\begin{array}{ccc}
\cos \theta_{1} & -\sin \theta_{1} & 0 \\
\sin \theta_{1} & \cos \theta_{1} & 0 \\
0 & 0 & 1
\end{array}\right] e^{X}\left[\begin{array}{ccc}
1 & 0 & 0 \\
0 & \cos \theta_{2} & -\sin \theta_{2} \\
0 & \sin \theta_{2} & \cos \theta_{2}
\end{array}\right] e^{Y}\left[\begin{array}{ccc}
\cos \theta_{3} & -\sin \theta_{3} & 0 \\
\sin \theta_{3} & \cos \theta_{3} & 0 \\
0 & 0 & 1
\end{array}\right]\right) \\
& =a\left(e^{X}\left[\begin{array}{ccc}
1 & 0 & 0 \\
0 & \cos \theta_{2} & -\sin \theta_{2} \\
0 & \sin \theta_{2} & \cos \theta_{2}
\end{array}\right] e^{Y}\right)
\end{aligned}
$$

(any $k \in \mathbf{S O}(3)$ can be written in that way). Hence, $a\left(e^{X} \mathbf{S O}(3) e^{Y}\right)$ has dimension at most 1 and therefore does not contain an open set.

Table 3 shows however that even in the low rank cases such as $B_{2}, A_{3}$, it is possible for the density to exist when both $X$ and $Y$ are singular.

We arrive in this way to the challenging Problem 2: characterize all pairs $X$, $Y$ of singular elements

$$
X, Y \notin W \cdot \mathfrak{a}^{+}
$$

such that the measure $m_{X, Y}=\delta_{e^{X}}^{\natural} \star \delta_{e^{Y}}^{\natural}$ has a density. It should depend on how irregular $X$ and $Y$ are.

In our most recent papers, we solved this problem for:

- classical symmetric spaces of type $A_{n}$,

- the symmetric spaces $\mathbf{S O}(p, q) / \mathbf{S O}(p) \times \mathbf{S O}(q), p \leq q$, containing (for $p<q)$ the noncompact Grassmanians,

- their complex and quaternionic analogs $\mathbf{S U}(p, q) / \mathbf{S U}(p) \times \mathbf{S U}(q)$ and $\mathbf{S p}(p, q) / \mathbf{S p}(p) \times \mathbf{S p}(q)$

- the spaces $\mathbf{S O}^{*}(2 n) / \mathbf{U}(n)$. 
TheOREm 2.6. In Table 2 and Table 3, we provide definitions of eligibility for a pair of elements of $\mathfrak{a}$ for the spaces $\mathbf{S L}(n, \mathbf{F}), \mathbf{F}=\mathbf{R}, \mathbf{C}, \mathbf{H}$ for all $n \geq 2, \mathbf{E}_{6} / \mathbf{F}_{4}$, $\mathbf{S O}(p, q) / \mathbf{S O}(p) \times \mathbf{S O}(q), \mathbf{S U}(p, q) / \mathbf{S U}(p) \times \mathbf{S U}(q), \mathbf{S p}(p, q) / \mathbf{S p}(p) \times \mathbf{S p}(q), p \leq q$ and $\mathbf{S O}^{*}(2 n) / \mathbf{U}(n), n \geq 2$. In all these cases, the measure $\mu_{X, Y}$ is absolutely continuous if an only if the pair $(X, Y)$ is eligible.

\begin{tabular}{|c|c|c|}
\hline Symmetric space & \multirow{2}{*}{$\begin{array}{l}\text { Description of } X \in \mathfrak{a}^{+} \\
\operatorname{diag}\left[x_{1}, \ldots, x_{n}\right], \\
\sum_{i=1}^{n} x_{i}=0, \\
x_{1}>x_{2}>\cdots>x_{n}\end{array}$} & Configuration of $X$ \\
\hline $\begin{array}{l}\mathbf{S L}(n, \mathbf{F}) / \mathbf{S U}(n, \mathbf{F}), \\
\mathbf{F}=\mathbf{R}, \mathbf{C}, \mathbf{H}, n \geq 2, \\
\mathbf{F}=\mathbf{O}, n=3 \\
\left(\text { i.e. } \mathbf{E}_{6} / \mathbf{F}_{4}\right)\end{array}$ & & $\begin{array}{l}X=X\left[s_{1}, \ldots, s_{r}\right], s_{i} \\
\text { number of repetitions of } \\
\text { the } x_{i} \text { 's }\end{array}$ \\
\hline $\begin{array}{l}\mathbf{S O}(p, q) / \mathbf{S O}(p) \times \mathbf{S O}(q), \\
p<q, \\
\mathbf{S U}(p, q) / \mathbf{S U}(p) \times \mathbf{S U}(q), \\
\mathbf{S p}(p, q) / \mathbf{S p}(p) \times \mathbf{S p}(q), \\
p \leq q\end{array}$ & $\begin{array}{l}{\left[\begin{array}{ccc}0 & \mathcal{D}_{X} & 0 \\
\mathcal{D}_{X} & 0 & 0 \\
0 & 0 & 0\end{array}\right]} \\
\text { in } \mathfrak{g l}(p+q, \mathbf{R}), \\
\mathcal{D}_{X}=\operatorname{diag}\left[x_{1}, \ldots, x_{p}\right], \\
x_{1}>x_{2}>\cdots>x_{p}>0\end{array}$ & $\begin{array}{l}X=X\left[s_{1}, \ldots, s_{r} ; u\right], s_{i} \\
\text { number of repetitions of } \\
\text { the nonzero } x_{i} \text { 's and } u \text { the } \\
\text { number of } x_{i}=0\end{array}$ \\
\hline $\mathbf{S O}(p, p) / \mathbf{S O}(p) \times \mathbf{S O}(p)$ & $\begin{array}{l}{\left[\begin{array}{cc}0 & \mathcal{D}_{X} \\
\mathcal{D}_{X} & 0\end{array}\right] \in \mathfrak{g l}(2 p, \mathbf{R}),} \\
\mathcal{D}_{X}=\operatorname{diag}\left[x_{1}, \ldots, x_{p}\right] \\
x_{1}>x_{2}>\cdots>x_{p-1}> \\
\left|x_{p}\right|\end{array}$ & $\begin{array}{l}X=X\left[s_{1}, \ldots, s_{r} ; u\right], s_{i} \\
\text { number of repetitions of } \\
\text { the nonzero }\left|x_{i}\right| \text { 's and } u \\
\text { the number of } x_{i}=0\end{array}$ \\
\hline $\mathbf{S O}^{*}(2 n) / \mathbf{U}(n)$ & $\begin{array}{l}{\left[\begin{array}{c|c}0 & \mathcal{E}_{X} \\
-\mathcal{E}_{X} & 0\end{array}\right] \in \mathfrak{g l}(2 n, \mathbf{R}),} \\
\mathcal{E}_{X}=\sum_{k=1}^{[n / 2]} x_{k} \\
\quad\left(E_{2 k, 2 k+1}-E_{2 k+1,2 k}\right), \\
x_{1}>x_{2}>\cdots>x_{n / 2}>0\end{array}$ & $\begin{array}{l}X=X\left[s_{1}, \ldots, s_{r} ; u\right], s_{i} \\
\text { number of repetitions of } \\
\text { the nonzero } x_{i} \text { 's and } u \text { the } \\
\text { number of } x_{i}=0\end{array}$ \\
\hline
\end{tabular}

TABle 2. Configurations of elements of $\mathfrak{a}$ 
CONVOLUTION OF ORBITAL MEASURES ON SYMMETRIC SPACES: A SURVEY 11

\begin{tabular}{|c|c|c|c|}
\hline Symmetric space & Eligibility & $\begin{array}{l}X \quad \neq 0 \\
\left(\delta_{e^{x}}^{\natural}\right)^{l} \\
\text { absolutely } \\
\text { contin- } \\
\text { uous } \\
\text { (optimal } \\
\text { value) }\end{array}$ & Reference \\
\hline $\begin{array}{l}\mathbf{S L}(n, \mathbf{F}) / \mathbf{S U}(n, \mathbf{F}) \\
\mathbf{F}=\mathbf{R}, \mathbf{C}, \mathbf{H}, n \geq 2, \\
\mathbf{F}=\mathbf{O}, n=3 \\
\left.\text { (i.e. } \mathbf{E}_{6} / \mathbf{F}_{4}\right)\end{array}$ & $\begin{array}{l}X=X[\mathbf{s}], Y=Y[\mathbf{t}]: \\
\max \{\mathbf{s}\}+\max \{\mathbf{t}\} \leq n, \\
\text { (and } X \text { or } Y \text { not of the form } \\
\left.a \operatorname{diag}\left[I_{n / 2},-I_{n / 2}\right]\right)\end{array}$ & $l \geq n$ & {$[9]$} \\
\hline $\begin{array}{l}\mathbf{S O}(p, q) / \mathbf{S O}(p) \times \mathbf{S O}(q), \\
p<q, \\
\mathbf{S U}(p, q) / \mathbf{S U}(p) \times \mathbf{S U}(q), \\
\mathbf{S p}(p, q) / \mathbf{S p}(p) \times \mathbf{S p}(q), \\
p \leq q\end{array}$ & $\begin{array}{l}X=X[\mathbf{s} ; u], Y=Y[\mathbf{t} ; v]: \\
\max (\mathbf{s}, 2 u)+\max (\mathbf{t}, 2 v) \leq 2 p\end{array}$ & $l \geq p$ & {$[7,8]$} \\
\hline $\mathbf{S O}(p, p) / \mathbf{S O}(p) \times \mathbf{S O}(p)$ & $\begin{array}{l}X=X[\mathbf{s} ; u], Y=Y[\mathbf{t} ; v]: \\
\text { if } u \leq 1, v \leq 1 \text { then } \\
\max (\mathbf{s})+\max (\mathbf{t}) \leq 2 p-2, \\
\text { if } u \geq 2 \text { or } v \geq 2 \text { then } \\
\max (\mathbf{s}, 2 u)+\max (\mathbf{t}, 2 v) \leq 2 p \\
\left(\text { if } p=4, \text { then }\left\{\mathcal{D}_{X}, \mathcal{D}_{Y}\right\}\right. \\
\neq\{\operatorname{diag}[a, a, a, a], \operatorname{diag}[b, b, c, c]\})\end{array}$ & $\begin{array}{l}l \geq p \text { if } \\
p \geq 4 \\
l \geq p+1 \\
\text { if } p=2,3\end{array}$ & {$[7]$} \\
\hline $\mathbf{S O}^{*}(2 n) / \mathbf{U}(n)$ & $\begin{array}{l}X=X[\mathbf{s} ; u], Y=Y[\mathbf{t} ; v]: \\
\max (\mathbf{s}, 2 u)+\max (\mathbf{t}, 2 v) \leq 2\left[\frac{n}{2}\right]\end{array}$ & $l \geq[n / 2]$ & {$[31]$} \\
\hline
\end{tabular}

TABLE 3. Summary of results

2.2.1. Comments on Problem 3. The last column of the last table concerns the absolute continuity of convolution powers.

Ragozin showed in $[\mathbf{2 9}]$ that for any Riemannian symmetric space $G / K$ and any $X_{j} \neq 0 \in \mathfrak{a}, j=1, \ldots, m$, the measure

$$
\delta_{e^{X_{1}}}^{\natural} \star \cdots \star \delta_{e^{x_{m}}}^{\natural}
$$

is absolutely continuous for $m \geq \operatorname{dim}(G / K)$.

In $[\mathbf{1 0}]$, we proved a much stronger property: under the same conditions, the measure in (2.3) is absolutely continuous for $m \geq r+1$, where $r$ is the rank of $G / K$. As can be seen from Table 3 , this bound cannot be improved since it is optimal for the root systems of type $A$.

THEOREM 2.7. Table 3 provides the optimal values $m$ which ensure that the convolution powers $\left(\delta_{e^{X}}^{\natural}\right)^{l}, X \neq 0$, are absolutely continuous for the spaces $\mathbf{S L}(n, \mathbf{F})$, $\mathbf{F}=\mathbf{R}, \mathbf{C}, \mathbf{H}$ for all $n \geq 2, \mathbf{E}_{6} / \mathbf{F}_{4}, \mathbf{S O}(p, q) / \mathbf{S O}(p) \times \mathbf{S O}(q), \mathbf{S U}(p, q) / \mathbf{S U}(p) \times$ $\mathbf{S U}(q), \mathbf{S p}(p, q) / \mathbf{S p}(p) \times \mathbf{S p}(q), p \leq q$ and $\mathbf{S O}^{*}(2 n) / \mathbf{U}(n), n \geq 2$. These values $m$ are optimal in the sens that there are choices of $X \neq 0$ for which $\left(\delta_{e^{X}}^{\natural}\right)^{l}$ is not absolutely continuous when $l<m$. We provide in Table 4 examples of such choices in each case. In a sense, these are the "most singular" elements of their respective Cartan subalgebra. 


\begin{tabular}{|l|l|c|}
\hline Symmetric space & \multicolumn{1}{|c|}{$X$} & $\left(\delta_{e^{X}}^{\natural}\right)^{l}$ not absolutely continuous \\
\hline $\mathbf{S L}(n, \mathbf{F}) / \mathbf{S U}(n, \mathbf{F})$, & $X=X[n-1,1]$ & $l<n$ \\
$\mathbf{F}=\mathbf{R}, \mathbf{C}, \mathbf{H}, n \geq 2$, & & \\
$\mathbf{F}=\mathbf{O}, n=3\left(i . e . \mathbf{E}_{6} / \mathbf{F}_{4}\right)$ & & $l<p$ \\
\hline $\begin{array}{l}\mathbf{S O}(p, q) / \mathbf{S O}(p) \times \mathbf{S O}(q), p<q, \\
\mathbf{S U}(p, q) / \mathbf{S U}(p) \times \mathbf{S U}(q),\end{array}$ & $X=X[1 ; p-1]$ & \\
$\mathbf{S p}(p, q) / \mathbf{S p}(p) \times \mathbf{S p}(q), p \leq q$ & & $l<p, p \geq 4$ \\
\hline $\mathbf{S O}(p, p) / \mathbf{S O}(p) \times \mathbf{S O}(p)$ & $X=X[1 ; p-1]$ & $l<p+1, p=2,3$ \\
\cline { 2 - 3 } & $X=X[p]$ & $l<[n / 2]$ \\
\hline $\mathbf{S O}^{*}(2 n) / \mathbf{U}(n)$ & $X=X[1 ;[n / 2]-1]$ & \\
\hline
\end{tabular}

TABLE 4. Convolutions powers which are not absolutely continuous

2.3. Proofs. We sketch the idea of the proof in the case of the spaces $\mathbf{S O}_{0}(p, q) / \mathbf{S O}(p) \times$ $\mathbf{S O}(q), p<q$. This is a good choice for illustration purposes: the proof for the symmetric spaces of type $A$ is simpler while the proof in the case of the spaces $\mathbf{S O}_{0}(p, p) / \mathbf{S O}(p) \times \mathbf{S O}(p)$ is more technical.

2.3.1. The case of the noncompact Grassmanians. In [7], we discussed the product formula on the spaces

$$
\mathbf{S O}_{0}(p, q) /(\mathbf{S O}(p) \times \mathbf{S O}(q)) \text { with } q>p .
$$

In what follows, with the help of selected examples, we will outline the techniques that led to the results in Table 3 . We start by presenting here the necessary information on the spaces.

We defined $\mathbf{S O}(p, q)$ as the group of matrices $g \in \mathbf{S L}(p+q, \mathbf{R})$ such that $g^{T} I_{p, q} g=I_{p, q}$ where $I_{p, q}=\left[\begin{array}{cc}-I_{p} & 0_{p \times q} \\ 0_{q \times p} & I_{q}\end{array}\right]$. Therefore the Lie algebra $\mathfrak{s o}(p, q)$ of $\mathbf{S O}_{0}(p, q)$ (the connected component of $\mathbf{S O}(p, q)$ ) consists of the matrices

$$
\left[\begin{array}{cc}
A & B \\
B^{T} & D
\end{array}\right]
$$

where $A$ and $D$ are skew-symmetric.

- The maximal compact subgroup $K=\left\{\left[\begin{array}{cc}A & 0 \\ 0 & D\end{array}\right]: A \in \mathbf{S O}(p), B \in \mathbf{S O}(q)\right\}$

$\bullet \mathfrak{p}=\left\{\left[\begin{array}{cc}0 & B_{p \times q} \\ B^{T} & 0\end{array}\right]\right\}$.

- The Cartan involution is given by $\theta(X)=-X^{T}$.

- The Cartan subalgebra consists of $\mathfrak{a}=\left\{H=\left[\begin{array}{ccc}0 & \mathcal{D}_{H} & 0_{p \times(q-p)} \\ \mathcal{D}_{H} & 0 & 0 \\ 0_{(q-p) \times p} & 0 & 0\end{array}\right]\right\}$ where $\mathcal{D}_{H}=\operatorname{diag}\left[H_{1}, \ldots, H_{p}\right]$.

We describe the root system of the Lie algebra $\mathfrak{s o}(p, q)$ in Table 5 .

The positive roots can be chosen as $\alpha(H)=H_{i} \pm H_{j}, 1 \leq i<j \leq p$ and $\alpha(H)=H_{i}, i=1, \ldots, p$ with simple $\operatorname{roots} \alpha_{i}(H)=H_{i}-H_{i+1}, i=1, \ldots, p-1$ and $\alpha_{p}(H)=H_{p}$. The positive Weyl chamber $\mathfrak{a}^{+}$consists therefore of $H \in \mathfrak{a}$ with 


\begin{tabular}{|c|c|c|}
\hline$\alpha$ & $m_{\alpha}$ & root vectors $X_{\alpha}$ \\
\hline $\pm H_{i}$ & $q-p$ & $X_{i r}^{ \pm}=E_{i 2 p+r}+E_{2 p+r i}$ \\
$1 \leq i \leq p$ & & $\begin{array}{c} \pm\left(E_{p+i 2 p+r}-E_{2 p+r p+i}\right) \\
r=1, \ldots, q-p\end{array}$ \\
& & $Y_{i j}^{ \pm}=E_{i p+j}+E_{p+j i}+E_{j p+i}+E_{p+i j}$ \\
$\pm\left(H_{i}-H_{j}\right)$ & 1 & $\pm\left(E_{i j}-E_{j i}+E_{p+i p+j}-E_{p+j p+i}\right)$ \\
$1 \leq i<j \leq p$ & & $Z_{i j}^{ \pm}=-\left(E_{i p+j}+E_{p+j i}\right)+E_{j p+i}+E_{p+i j}$ \\
$\pm\left(E_{i j}-E_{j i}-E_{p+i p+j}+E_{p+j p+i}\right)$ \\
\hline $1 \leq i<j \leq p$
\end{tabular}

TABLE 5. Restricted roots and associated root vectors

$\mathcal{D}_{H}=\operatorname{diag}\left(H_{1}, \ldots, H_{p}\right)$ such that

$$
H_{1}>H_{2}>\cdots>H_{p}>0 .
$$

The elements of the Weyl group $W$ are permutations of the diagonal entries of $\mathcal{D}_{X}$ with eventual sign changes of any number of these entries.

We need to describe how irregular an element of $\mathfrak{a}$ is: let $X \in \overline{\mathfrak{a}^{+}}$. We will write $X=X[\mathbf{s} ; u]$ if $X=\left[\begin{array}{ccc}0 & \mathcal{D}_{X} & 0_{p \times(q-p)} \\ \mathcal{D}_{X} & 0 & 0 \\ 0_{(q-p) \times p} & 0 & 0\end{array}\right]$ with

$$
\mathcal{D}_{X}=\operatorname{diag}[\overbrace{x_{1}, \ldots, x_{1}}^{s_{1} \geq 1}, \overbrace{x_{2}, \ldots, x_{2}}^{s_{2} \geq 1}, \ldots, \overbrace{x_{M}, \ldots, x_{M}}^{s_{M} \geq 1}, \overbrace{0 \ldots, 0}^{u \geq 0}] .
$$

If $X \notin \overline{\mathfrak{a}^{+}}$then we will associate to $X$ the configuration of $w \cdot X \in \overline{\mathfrak{a}^{+}}$.

Definition 2.8. Let $X=X[\mathbf{s} ; u]$ and $Y=Y[\mathbf{t} ; v]$. We say that $X$ and $Y$ are eligible if

$$
\max \{\mathbf{s}, 2 u\}+\max \{\mathbf{t}, 2 v\} \leq 2 p .
$$

Theorem 2.9. Let $G=\mathbf{S O}_{0}(p, q)$ and let $X, Y \in \mathfrak{a}$. Then the measure $m_{X, Y}$ is absolutely continuous if and only if $X$ and $Y$ are eligible.

Proof. We now sketch the proof in the case $\mathbf{S O}_{0}(p, q) / \mathbf{S O}(p) \times \mathbf{S O}(q)$. We start by showing that the eligibility is a sufficient condition.

Our proof uses the criterion developed in Theorem 2.3, part (7). It follows the following steps.

(1) Proof for $p=1, q=2$.

In [8], our starting point was $p=2, q=3$ but that was unnecessarily complicated. If we start at $p=1$, one only needs to notice that we either have $X=0$ or $X \in \mathfrak{a}^{+}$(similarly for $Y$ ).

(2) Proof in the case $q=p+1$ using induction on $p$.

(a) For $X=X[\mathbf{s} ; u]$ and $Y=Y[\mathbf{t} ; v]$ with $u>0$ or $v>0$.

(b) For $X=X[\mathbf{s} ; 0]$ and $Y=Y[\mathbf{t} ; 0]$.

In both cases, instead of the full proof, we will provide illustrative examples.

(3) Proof that $(p, p+1) \Rightarrow(p, q)$ for $q>p+1$.

Again the proof given in [8] of this step can be simplified. It suffices to notice that if the $(2 p+1) \times(2 p+1)$ submatrices $X^{\prime}$ and $Y^{\prime}$ of $X$, 
$Y \in \mathfrak{s o}(p, q)$ are such that $\mathcal{D}_{X^{\prime}}=\mathcal{D}_{X}$ and $\mathcal{D}_{Y^{\prime}}=\mathcal{D}_{Y}$ and using $\mathbf{S O}(p) \times$ $\mathbf{S O}(p+1) \simeq \mathbf{S O}(p) \times \mathbf{S O}(p+1) \times\left\{I_{q-(p+1)}\right\}$, then

$$
\begin{aligned}
a\left(e^{X^{\prime}} \mathbf{S O}(p) \times \mathbf{S O}(p+1) e^{Y^{\prime}}\right) & \simeq a\left(e^{X} \mathbf{S O}(p) \times \mathbf{S O}(p+1) \times\left\{I_{q-(p+1)}\right\} e^{Y}\right) \\
& \subset a\left(e^{X} \mathbf{S O}(p) \times \mathbf{S O}(q) e^{Y}\right) ;
\end{aligned}
$$

Therefore if the left hand side has an open set homeomorphic to an open set of $\mathbf{R}^{p}$, the same has to be true of the rightmost side.

Sketch of the proof of the case $X=X[\mathbf{s} ; u]$ and $Y=Y[\mathbf{t} ; v]$ with $u>0$ or $v>0$ via an example $(p=3$ and $q=4)$.

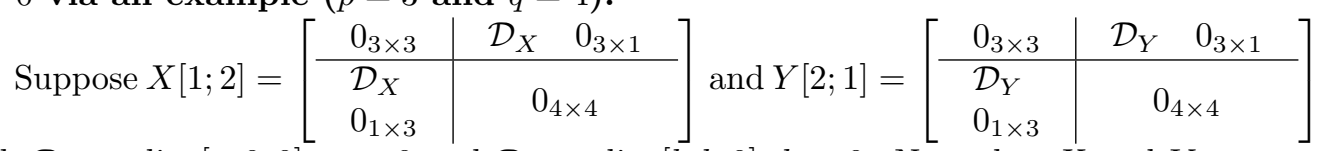

with $\mathcal{D}_{X}=\operatorname{diag}[a, 0,0], a>0$ and $\mathcal{D}_{Y}=\operatorname{diag}[b, b, 0], b>0$. Note that $X$ and $Y$ are eligible: $2 \cdot 2+2 \leq 2 \cdot 3$.

The induction step: $\mathcal{D}_{X}=\operatorname{diag}[0, \overbrace{a, 0}^{X^{\prime}}]$ and $\mathcal{D}_{Y}=\operatorname{diag}[b, \overbrace{b, 0}^{Y^{\prime}}]: X^{\prime}=X^{\prime}[1 ; 1]$ and $Y^{\prime}=Y^{\prime}[1 ; 1]$ are eligible: $2+2 \cdot 1 \leq 2 \cdot 2$. There is a method in choosing "good" predecessor $X^{\prime}$ and $Y^{\prime}$ : we placed one of the zeros of $\mathcal{D}_{X}$ at the start and the rest at the end while we ensured that the largest block of $\mathcal{D}_{Y}$ was at the start. The construction of $X^{\prime}$ and $Y^{\prime}$ is then as shown in all cases.

By induction, there exists $k_{0}^{\prime}=\left[\begin{array}{cc}A & 0 \\ 0 & B\end{array}\right]$ such that $V_{X^{\prime}}+\operatorname{Ad}\left(k_{0}^{\prime}\right) V_{Y^{\prime}}=\mathfrak{p}^{\prime}$ or

$$
V_{X^{\prime}}+\operatorname{Ad}\left(k_{0}\right) V_{Y^{\prime}}=\left[\begin{array}{ccc|cccc}
0 & 0 & 0 & 0 & 0 & 0 & 0 \\
0 & 0 & 0 & 0 & * & * & * \\
0 & 0 & 0 & 0 & * & * & * \\
\hline 0 & 0 & 0 & 0 & 0 & 0 & 0 \\
0 & * & * & 0 & 0 & 0 & 0 \\
0 & * & * & 0 & 0 & 0 & 0 \\
0 & * & * & 0 & 0 & 0 & 0
\end{array}\right]
$$

with $k_{0}=\left[\begin{array}{cccc}1 & 0 & 0 & 0 \\ 0 & A & 0 & 0 \\ 0 & 0 & 1 & 0 \\ 0 & 0 & 0 & B\end{array}\right]$. Now, $V_{X}=\operatorname{span}(\overbrace{\left\{Z_{12}, Y_{12}\right\}}^{N_{X}} \cup V_{X^{\prime}}), V_{Y}=\operatorname{span}(\overbrace{\left\{X_{1}, Z_{12}, Z_{13}, Y_{13}\right\}}^{N_{Y}} \cup V_{Y^{\prime}})$.

By straightforward computations, we find that

$$
\begin{gathered}
\operatorname{Ad}\left(k_{0}\right)\left(\operatorname{span}\left(N_{Y}\right)\right) \\
=\operatorname{span}\left\{\operatorname{Ad}\left(k_{0}\right) X_{1}=\left[\begin{array}{c|c}
0 & \beta_{3}^{T} \\
\hline 0 & 0
\end{array}\right]^{s}, \operatorname{Ad}\left(k_{0}\right) Z_{12}=\left[\begin{array}{c|c}
0 & \beta_{1}^{T} \\
\hline-\alpha_{1} & 0
\end{array}\right]^{s},\right. \\
\left.\operatorname{Ad}\left(k_{0}\right) Z_{13}=\left[\begin{array}{c|c|c}
0 & \beta_{2}^{T} \\
\hline-\alpha_{2} & 0
\end{array}\right]^{s}, \operatorname{Ad}\left(k_{0}\right) Y_{13}=\left[\begin{array}{c|c}
0 & \beta_{2}^{T} \\
\hline \alpha_{2} & 0
\end{array}\right]^{s}\right\}
\end{gathered}
$$

where the $\alpha_{i}$ 's are the columns of $A$ and the $\beta_{i}$ 's the columns of $B$. Given that $A$ and $B$ are non-singular, it is easy to see that these matrices are linearly independent. We want to have

$$
\operatorname{Ad}\left(k_{0}\right)\left(\operatorname{span}\left(N_{Y}\right)\right)=\left[\begin{array}{ccc|cccc}
0 & 0 & 0 & 0 & a_{1} & a_{2} & a_{3} \\
0 & 0 & 0 & \tau & 0 & 0 & 0 \\
0 & 0 & 0 & a_{4} & 0 & 0 & 0 \\
\hline 0 & \tau & a_{4} & 0 & 0 & 0 & 0 \\
a_{1} & 0 & 0 & 0 & 0 & 0 & 0 \\
a_{2} & 0 & 0 & 0 & 0 & 0 & 0 \\
a_{3} & 0 & 0 & 0 & 0 & 0 & 0
\end{array}\right]
$$

where the $a_{i}$ 's are arbitrary and $\tau$ depends on the $a_{i}$ 's. This is possible as long as the second component of $\alpha_{2}$ is not zero. Since this is the case for a dense open 
subset of $\mathbf{S O}(2)$ and (2.4) is valid for a dense open subset of $\mathbf{S O}(2) \times \mathbf{S O}(3)$, we can assume that this is the case (recall Remark 2.2). Hence, we have

$$
V_{X^{\prime}}+\operatorname{Ad}\left(k_{0}\right) \overbrace{\operatorname{span}\left\{N_{Y} \cup V_{Y^{\prime}}\right\}}^{V_{Y}})=\left[\begin{array}{ccc|cccc}
0 & 0 & 0 & 0 & a_{1} & * & * \\
0 & 0 & 0 & \tau & * & * & * \\
0 & 0 & 0 & * & * & * & * \\
\hline 0 & \tau & * & 0 & 0 & 0 & 0 \\
a_{1} & * & * & 0 & 0 & 0 & 0 \\
* & * & * & 0 & 0 & 0 & 0 \\
* & * & * & 0 & 0 & 0 & 0
\end{array}\right]
$$

where only the entry $\tau$ is not arbitrary. If $\tau=a_{1}$, then we pick $Z_{1,2}$ from $N_{X}$, if $\tau=-a_{1}$, we pick $Y_{1,2}$ otherwise, we pick either. To fix things, suppose that we pick $Y_{1,2}$. Then

$$
\operatorname{span}\left\{Y_{1,2}\right\} \oplus V_{X^{\prime}}+\operatorname{Ad}\left(k_{0}\right)\left(V_{Y}\right)=\left[\begin{array}{ccc|cccc}
0 & 0 & 0 & 0 & * & * & * \\
0 & 0 & 0 & * & * & * & * \\
0 & 0 & 0 & * & * & * & * \\
\hline 0 & * & * & 0 & 0 & 0 & 0 \\
* & * & * & 0 & 0 & 0 & 0 \\
* & * & * & 0 & 0 & 0 & 0 \\
* & * & * & 0 & 0 & 0 & 0
\end{array}\right] .
$$

Next we note that for $t>0$ small enough

$$
\begin{aligned}
& \operatorname{Ad}\left(e^{t\left(Z_{12}^{+}+\theta Z_{12}^{+}\right)}\right)\left(\operatorname{span}\left\{Y_{1,2}\right\} \oplus V_{X^{\prime}}\right)+\operatorname{Ad}\left(k_{0}\right)\left(V_{Y}\right) \\
& =\left[\begin{array}{lll|llll}
0 & 0 & 0 & 0 & * & * & * \\
0 & 0 & 0 & * & * & * & * \\
0 & 0 & 0 & * & * & * & * \\
\hline 0 & * & * & 0 & 0 & 0 & 0 \\
* & * & * & 0 & 0 & 0 & 0 \\
* & * & * & 0 & 0 & 0 & 0 \\
* & * & * & 0 & 0 & 0 & 0
\end{array}\right]
\end{aligned}
$$

(for $t$ small enough the dimension of this space remains the same; the position in upper left angle of $\mathfrak{p}$ is not affected).

Finally, we use the last vector $Z_{1,2}$ from $N_{X}$ :

$$
\begin{aligned}
& \left.\operatorname{Ad}\left(e^{t\left(Z_{12}^{+}+\theta\right.} Z_{12}^{+}\right)\right) \\
& =\left[\begin{array}{ccc|cccc}
0 & 0 & 0 & * & * & * & * \\
0 & 0 & 0 & * & * & * & * \\
0 & 0 & 0 & * & * & * & * \\
\hline * & * & * & 0 & 0 & 0 & 0 \\
* & * & * & 0 & 0 & 0 & 0 \\
* & * & * & 0 & 0 & 0 & 0 \\
* & * & * & 0 & 0 & 0 & 0
\end{array}\right]=\mathfrak{p} \\
& V_{X}
\end{aligned}
$$

if $t$ is close to 0 since $\operatorname{Ad}\left(e^{t\left(Z_{12}^{+}+\theta Z_{12}^{+}\right)}\right)\left(Z_{12}\right)=\cos (4 t) Z_{12}+2 \sin (4 t)\left(A_{1}+A_{2}\right)$. Therefore,

$$
V_{X}+\operatorname{Ad}(\overbrace{e^{-t\left(Z_{12}^{+}+\theta Z_{12}^{+}\right)} k_{0}}^{k}) V_{Y}=\operatorname{Ad}\left(e^{-t\left(Z_{12}^{+}+\theta Z_{12}^{+}\right)}\right) \mathfrak{p}=\mathfrak{p}
$$

which means that the density exists.

Sketch of the proof of the case with $u=0$ and $v=0$ via an example ( $p=3$ and $q=4)$.

If $=0$ and $v=0$, we can assume that $X=X[p]$ and $Y=Y[p]$. Indeed, For all the $X$ and $Y$ with $u=v=0$, this choice is the one with the smallest $V_{X}$ and $V_{Y}$. In our particular example, $\mathcal{D}_{X}=\operatorname{diag}[a, \overbrace{a, a}^{X^{\prime}}]$ and $\mathcal{D}_{Y}=[b, \overbrace{b, b}^{Y^{\prime}}]$ with $a>0$ and $b>0$. Assuming that the case $p=2$ has been solved, we can know that there 
exists $k_{0}^{\prime}=\left[\begin{array}{cc}A & 0 \\ 0 & B\end{array}\right]$ such that $V_{X^{\prime}}+\operatorname{Ad}\left(k_{0}^{\prime}\right) V_{Y^{\prime}}=\mathfrak{p}^{\prime}$ or

$$
V_{X^{\prime}}+\operatorname{Ad}\left(k_{0}\right) V_{Y^{\prime}}=\left[\begin{array}{ccc|cccc}
0 & 0 & 0 & 0 & 0 & 0 & 0 \\
0 & 0 & 0 & 0 & * & * & * \\
0 & 0 & 0 & 0 & * & * & * \\
\hline 0 & 0 & 0 & 0 & 0 & 0 & 0 \\
0 & * & * & 0 & 0 & 0 & 0 \\
0 & * & * & 0 & 0 & 0 & 0 \\
0 & * & * & 0 & 0 & 0 & 0
\end{array}\right]
$$

with $k_{0}=\left[\begin{array}{cccc}1 & 0 & 0 & 0 \\ 0 & A & 0 & 0 \\ 0 & 0 & 1 & 0 \\ 0 & 0 & 0 & B\end{array}\right]$. Now, $V_{X}=\operatorname{span} \overbrace{\left\{X_{1}, Z_{12}, Z_{13}\right\}}^{N_{X}}+V_{X^{\prime}}, V_{Y}=\operatorname{span} \overbrace{\left\{X_{1}, Z_{12}, Z_{13}\right\}}^{N_{Y}}+V_{Y^{\prime}}$.

As before, we find that

$$
\begin{aligned}
& \operatorname{Ad}\left(k_{0}\right)\left(\operatorname{span}\left(N_{Y}\right)\right)=\operatorname{span}\left\{\operatorname{Ad}\left(k_{0}\right) X_{1}=\left[\begin{array}{c|c}
0 & \beta_{3}^{T} \\
\hline 0 & 0
\end{array}\right]^{s}, A d\left(k_{0}\right) Z_{12}=\left[\begin{array}{c|c}
0 & \beta_{1}^{T} \\
\hline-\alpha_{1} & 0
\end{array}\right]^{s},\right. \\
& \left.\quad \operatorname{Ad}\left(k_{0}\right) Z_{13}=\left[\begin{array}{c|c}
0 & \beta_{2}^{T} \\
\hline-\alpha_{2} & 0
\end{array}\right]^{s}\right\} .
\end{aligned}
$$

Suppose for an instant that $A=-I_{2}$ and $B=I_{3}$. The matrices in (2.6) together with the matrices $Z_{1,2}$ and $Z_{1,3}$ are clearly linearly independent. By Remark 2.2, the set of matrices $k_{0}$ for which this holds is dense in $\mathbf{S O}(2) \times \mathbf{S O}(3)$. The same is true for the set of matrices for which (2.5) holds. By another application of Remark 2.2 , we can assume that both properties hold. We therefore have

$$
\left.\operatorname{span}\left\{Z_{1,2}, Z_{1,3}\right\} \oplus V_{X^{\prime}}+\operatorname{Ad}\left(k_{0}\right) V_{Y}\right)=\left[\begin{array}{ccc|cccc}
0 & 0 & 0 & 0 & * & * & * \\
0 & 0 & 0 & * & * & * & * \\
0 & 0 & 0 & * & * & * & * \\
\hline 0 & * & * & 0 & 0 & 0 & 0 \\
* & * & * & 0 & 0 & 0 & 0 \\
* & * & * & 0 & 0 & 0 & 0 \\
* & * & * & 0 & 0 & 0 & 0
\end{array}\right] .
$$

The remaining step is the same using $X_{1} \in N_{X}$ instead of $Z_{1,2}$ and $k_{t}=$ $e^{t\left(X_{1}^{+}+\theta\left(X_{1}^{+}\right)\right.}$.

To prove the necessity of the eligibility condition for the measures to be absolutely continuous we use similar approaches in all cases. We first introduce a matrix $S$ which allows us to jointly diagonalize every elements of $\mathfrak{a}$.

$$
\text { Let } J_{p}=\left(\delta_{i, p+1-i}\right) \in \mathfrak{g l}(p, \mathbf{R}) \text { and let } S=\left[\begin{array}{ccc}
\frac{\sqrt{2}}{2} I_{p} & 0_{p \times(q-p)} & \frac{\sqrt{2}}{2} J_{p} \\
\frac{\sqrt{2}}{2} I_{p} & 0_{p \times(q-p)} & -\frac{\sqrt{2}}{2} J_{p} \\
0_{(q-p) \times p} & I_{q-p} & 0_{(q-p) \times p}
\end{array}\right] \in
$$

$\mathbf{S O}(p+q)$. We check easily that $S^{T} H S=\operatorname{diag}[H_{1}, \ldots, H_{p}, \overbrace{0, \ldots, 0}^{q-p},-H_{p}, \ldots,-H_{1}]$ where, as before, $\mathcal{D}_{H}=\operatorname{diag}\left[H_{1}, \ldots, H_{p}\right]$.

This can be used to recover the diagonal part $\mathcal{D}_{H}$ of $H \in a\left(e^{X} K e^{Y}\right)$ from $a_{\mathbf{S L}(p+q)}(\overbrace{\left(S^{T} e^{X} S\right)}^{e^{S^{T}} X S} \overbrace{\left(S^{T} K S\right)}^{\subset \mathbf{S O}(p+q)} \overbrace{\left(S^{T} e^{Y} S\right)}^{e^{S^{T}} Y S})$.

The other device we use is given by Lemma 2.1. Suppose now that $X[\mathbf{s}, u], Y[\mathbf{t}, v] \in$ $\mathfrak{a}$ are not eligible: $\max \{\mathbf{s}, 2 u\}+\max \{\mathbf{t}, 2 v\}>2 p$ and apply Lemma 2.1 to $\tilde{H}=$ $a_{\mathbf{S L}(p+q)}\left(e^{S^{T} X S} \mathbf{S O}(p+q) e^{S^{T} Y S}\right)$.

There are essentially two cases:

- If $u+v>p$ then for any $H \in a\left(e^{X} K e^{Y}\right)$, the diagonal of $\mathcal{D}_{H}$ has $r+s-N=(2 u+q-p)+(2 v+q-p)-(p+q)=2(u+v-p)+(q-p)$ 
repetitions of $0+0=0$ which implies that $a\left(e^{X} K e^{Y}\right)$ has an empty interior.

- If $2 u+\overbrace{\max \{\mathbf{t}\}}^{t}>2 p$ and $Y_{i} \neq 0$ is repeated $t$ times then for any $H \in$ $a\left(e^{X} K e^{Y}\right)$, the diagonal of $\mathcal{D}_{H}$ has $r-s=r+(N-s)-N=(2 u+q-$ $p)+t-(p+q)=2 u+t-2 p>0$ repetitions of $Y_{i}+0 \neq 0$ which implies that $a\left(e^{X} K e^{Y}\right)$ has an empty interior.

We should note that the same result holds for the spaces $\mathbf{S U}(p, q) / \mathbf{S}(\mathbf{U}(p) \times$ $\mathbf{U}(q))$ and $\mathbf{S p}(p, q) / \mathbf{S p}(p) \times \mathbf{S p}(q), q>p$. Indeed, the same proof that the condition of eligibility is necessary applies directly to these spaces while we can directly embed the set $a\left(e^{X}(\mathbf{S O}(p) \times \mathbf{S O}(q)) e^{K}\right)$ for the space $\mathbf{S O}_{0}(p, q) / \mathbf{S O}([p) \times \mathbf{S O}(q)$ into the corresponding sets $a\left(e^{X}(\mathbf{S U}(p) \times \mathbf{S U}(q)) e^{K}\right)$ and $a\left(e^{X}(\mathbf{S p}(p) \times \mathbf{S p}(q)) e^{K}\right)$ of the spaces $\mathbf{S U}(p, q) / \mathbf{S}(\mathbf{U}(p) \times \mathbf{U}(q))$ and $\mathbf{S p}(p, q) / \mathbf{S p}(p) \times \mathbf{S p}(q)$.

2.3.2. Convolution powers. We will outline here how our previous results allow us to prove Theorem 2.7. Again, for illustrations purposes, we will provide the proof for one of the spaces, namely $\mathbf{S L}(n, \mathbf{F}) / \mathbf{S U}(n, \mathbf{F})$.

Proof. We wish to prove that for $X \neq 0,\left(\delta_{e^{x}}^{\natural}\right)^{l}$ is absolutely continuous for $l \geq n$ in the case of the root system $A_{n-1}$ (Problem 3). We will first show that this result cannot be improved. Indeed, consider $X=X[n-1,1]$ i.e. $X=$ $\operatorname{diag}[\overbrace{a, \ldots, a}^{n-1},-a], a>0$. Observe that the support of the measure $\left(\delta_{e^{X}}^{\natural}\right)^{l}$ is $\mathcal{S}_{r}^{X}=$ $a\left(e^{X} K e^{X} \ldots K e^{X}\right)$ where $K$ is repeated $l-1$ times. Using Lemma $2.1, l-1$ times, one notes that for every $H \in \mathcal{S}_{r}^{X}, \mathcal{D}_{H}$ has $n-l$ diagonal entries equal to $l a$. Therefore, $\mathcal{S}_{r}^{X}$ has an empty interior whenever $l<n$.

We now show that for $X \neq 0,\left(\delta_{e^{X}}^{\natural}\right)^{l}$ is absolutely continuous for $l \geq n$. It is enough to prove the following claim: $\mathcal{S}_{n-1}^{X} \cap \mathfrak{a}^{+} \neq \emptyset$. Indeed, if $H \in \mathcal{S}_{n-1}^{X} \cap \mathfrak{a}^{+}$then $a\left(e^{H} K e^{X}\right)$ has nonempty interior and the result follows since $a\left(e^{H} K e^{X}\right) \subset \mathcal{S}_{n}^{X}$.

We now prove the claim using induction on $n \geq 2$. When $n=2$ then $\mathcal{S}_{2}^{X}=a\left(e^{X} K e^{X}\right)$ has nonempty interior since in this case $X \in \mathfrak{a}^{+}$and therefore it intersects $\mathfrak{a}^{+}$. Suppose now the result true for $n-1$ and consider $\mathcal{S}_{n-2}^{X}$ : there exists then $H_{0}=\mathcal{S}_{n-2}^{X}$ with at least $n-1$ distinct elements on its diagonal and therefore, $H_{0}=H_{0}\left[1^{n-2}, 2\right]$ or $H_{0}=H_{0}\left[1^{n}\right]$. In the second case, we are done since $\mathcal{S}_{n-2}^{X} \subset \mathcal{S}_{n-1}^{X}$.

If $H_{0}=H_{0}\left[1^{n-2}, 2\right] \in \mathcal{S}_{n-2}^{X}$, we can assume that the diagonal entries which are identical in $\mathcal{D}_{X}$ and in $\mathcal{D}_{H_{0}}$ are at the end. We note that $X$ and $H_{0}$ considered without their last entries are eligible in $\mathbf{S L}(n-1, \mathbf{F})$ since the shortened $H_{0}$ is in $\mathfrak{a}^{+}$. Hence $a\left(e^{X} K_{0} e^{H_{0}}\right)$ has nonempty interior in the projection of the subspace $\overline{\mathfrak{a}^{+}}$ by removing the last row and column. Therefore, there exists $H \in a\left(e^{X} K_{0} e^{H_{0}}\right) \subset$ $S_{X}^{p-1}$ with $H=H\left[1^{n}\right]$ which proves the claim.

In order to solve the Problem 2 for all classical symmetric spaces of noncompact type, we still need to consider the spaces

$\mathbf{S p}(n, \mathbf{R}) / \mathbf{U}(n), \mathbf{S O}(2 n+1, \mathbf{C}) / \mathbf{S O}(2 n+1), \mathbf{S O}(2 n, \mathbf{C}) / \mathbf{S O}(2 n)$ and $\mathbf{S p}(n, \mathbf{C}) / \mathbf{S p}(n)$

as can be seen from $[\mathbf{1 6}$, Tables IV, V, Chapter X]. 


\section{Properties of the density of the convolution of orbital measures}

Once we know that the measures $m_{X, Y}$ and $\mu_{X, Y}$ are absolutely continuous, it is natural to study the properties of the corresponding density.

These questions are studied in $[\mathbf{1 1}, \mathbf{1 2}, \mathbf{1 4}]$ in the case of the measure $\mu_{X, Y}$ on $\overline{\mathfrak{a}^{+}}$or, equivalently, for its $W$-invariant version on the space $\mathfrak{a}$

$$
\mu_{X, Y}^{W}=\frac{1}{|W|} \sum_{w \in W} \mu_{X, Y} \circ w^{-1}
$$

Recall that the integration on $G$ written in polar coordinates, with suitable normalization, is given as

$$
\int_{G} f(g) d g=\int_{K} \int_{K} \int_{\mathfrak{a}^{+}} f\left(k_{1} e^{H} k_{2}\right) \delta(H) d H d k_{1} d k_{2}
$$

where $\delta(H)=\prod_{\alpha \in \Sigma^{+}} \sinh ^{m_{\alpha}} \alpha(H)$ and $m_{\alpha}$ denotes the multiplicity of the root $\alpha$. Hence $\delta$ is the density of the invariant measure on $\mathfrak{a}$ in polar coordinates i.e.

$$
\int_{G} h(g) d g=\int_{\mathfrak{a}^{+}} h\left(e^{H}\right) \delta(H) d H
$$

for any $K$-biinvariant function $h$ integrable on $G$.

It is thus relevant to study the density of the measures $\mu_{X, Y}$ or $\mu_{X, Y}^{W}$ with respect to the measure $\delta(H) d H$. We denote these two densities, respectively, by $k(H, X, Y)$ and $k^{W}(H, X, Y)$. Their supports

$$
S_{X, Y}=a\left(e^{X} K e^{Y}\right)=\operatorname{supp}\left(\mu_{X, Y}\right), S_{X, Y}^{W}=W \cdot a\left(e^{X} K e^{Y}\right)=\operatorname{supp}\left(\mu_{X, Y}^{W}\right)
$$

are compact since $S_{X, Y}$ is the continuous image of the compact group $K$.

3.1. Support. The results on the support contained in this section are valid for $X, Y \in \mathfrak{a}$.

Helgason [17, Prop. 10.13, Chapter IV] proved, using the celebrated Kostant convexity theorem, that

$$
S_{X, Y}^{W} \subset C(X)+C(Y)
$$

where $C(X)$ is the convex hull of the set $W X$.

We gave much more information on the support $S_{X, Y}$ in $[\mathbf{1 1}, \mathbf{1 2}, \mathbf{1 4}]$. We describe a "skeleton" set $I$ such that its convex hull equals $S_{X, Y}$.

Definition 3.1. Suppose $\alpha_{1}, \ldots, \alpha_{r}$ are the simple positive roots. For each $i$, let $\mathfrak{k}^{(i)}$ be the smallest Lie subalgebra of $\mathfrak{k}$ containing all the vectors $X_{\alpha}^{\mathfrak{k}}=X_{\alpha}+\theta X_{\alpha}$ with $X_{\alpha} \in \mathfrak{g}_{\alpha_{j}}$ and $j \neq i$. Let $K^{(i)}$ be the corresponding connected subgroup of $K$ and let

$$
K_{0}=\cup_{i=1}^{r} W K^{(i)} W
$$

For $X, Y \in \overline{\mathfrak{a}^{+}}$, we define the "skeleton" set $I$ by

$$
I=a\left(e^{X} K_{0} e^{Y}\right) .
$$

Of course $I \subset S_{X, Y}$. Figure 2 shows an example of the set $I$ in the rank 2 case $\mathbf{S L}(3, \mathbf{F}) / \mathbf{S U}(3, \mathbf{F})$. It may be then constructed from the hexagon $\partial(X+C(Y))$ together with its main diagonals, using the projection on the positive Weyl chamber. The details of this construction were provided in [12, Prop. 15].

The "skeleton" sets $K_{0}$ and $I$ are closely related to the surjectivity approach from Section 2. Indeed, we proved in [11, Cor. 2.13] that if $k \notin K_{0}$ and $a\left(e^{X} k e^{Y}\right) \in$ 

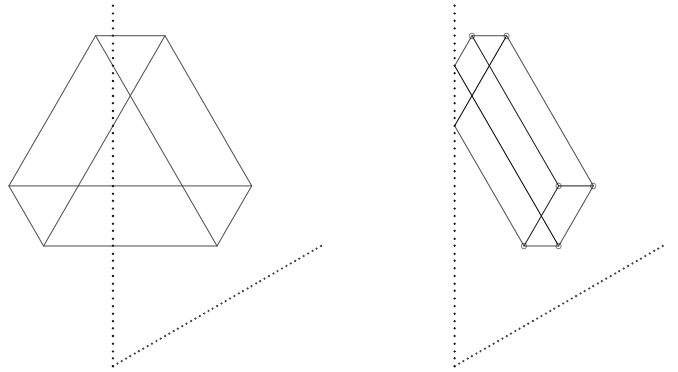

Figure 2. $X+C(Y)$ and $I$

$\mathfrak{a}^{+}$then the differential of the map $k \rightarrow a\left(e^{X} k e^{Y}\right)$ is surjective and $a\left(e^{X} k e^{Y}\right) \in$ int $S_{X, Y}$.

In the case of the spaces $\mathbf{S L}(3, \mathbf{F}) / \mathbf{S U}(3, \mathbf{F})$, we proved in $[\mathbf{1 2}$, Th. 25$]$ that

$$
S_{X, Y}=\operatorname{conv}(I) \text {. }
$$

In particular, we obtained that the set $S_{X, Y}$ is convex. Note that if $\mathbf{F}=\mathbf{C}$, the convexity of $S_{X, Y}$ is a consequence of [14, Prop.4.4], which says that on $\mathbf{S L}(3, \mathbf{C}) / \mathbf{S U}(3)$

$$
S_{X, Y}=(C(X)+Y) \cap(C(Y)+X) \cap\left\{H \in \overline{\mathfrak{a}^{+}} \mid H_{3} \leq X_{2}+Y_{2} \leq H_{1}\right\} .
$$

The last formula allowed us to produce the first images of the support of $k^{W}$ as shown in Figure 3.

$$
-7.0], Y=[3.0,1.0,-4.0] \quad-7.0], Y=[3.0,-1.0,-2.0]
$$
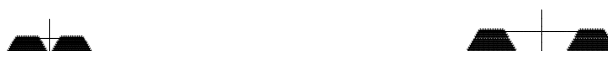

FiguRE 3. The support of $\mu_{X, Y}^{W}$

Several years later, the convexity of $S_{X, Y}$ was proved for all Riemannian symmetric spaces of noncompact type in [25]. It is a challenging problem to prove this convexity result by the methods of spherical harmonic analysis. Using the convexity of $S_{X, Y}$, we proved in [11, Th. 2.14] that

$$
S_{X, Y}=\operatorname{conv}(I)
$$

on all Riemannian symmetric spaces of noncompact type. 
3.2. Explicit formulas for the density. Explicit formulas for the function $k(H, X, Y)$ for the rank one case were given by Koornwinder in [23]. These formulas were derived using an addition formula for Jacobi functions, a tool that is not available in higher rank situations.

In [14], we exploited the particularly simple form of the spherical functions in the complex case (see for instance $[\mathbf{1 7}$, Th. 5.7, Chapter IV]) to give an explicit expression for the density of $\mu_{X, Y}$ when $X, Y \in \mathfrak{a}^{+}$. Recall that the kernel $K(X, H)$ of the Abel transform is defined by

$$
\phi_{\lambda}\left(e^{X}\right)=\int_{C(X)} e^{<i \lambda, H>} K(X, H) d H .
$$

In $\left[\mathbf{1 4}\right.$, Th. 2.1] we obtained the following expression for the density $k^{W}$ in the complex case:

$$
k^{W}(H, X, Y)=\frac{1}{\delta^{1 / 2}(H) \delta^{1 / 2}(Y)} \frac{1}{|W|} \sum_{w \in W} \operatorname{det}(w) K(X, w \cdot H-Y) .
$$

Let $\Sigma_{0}^{+}$be the set of positive roots, $\pi(\lambda)=\prod_{\alpha \in \Sigma_{0}^{+}}\langle\alpha, \lambda\rangle$ and $\rho$ the half-sum of the positive roots. Graczyk and Loeb had shown in [5] that

$$
K(X, H)=\frac{\pi(\rho)}{\delta^{1 / 2}(X)} \sum_{w \in W} \epsilon(w) T(w X-H) .
$$

where the function $T$ is defined by

$$
\int_{\mathbf{R}_{+}^{q}} f\left(\sum_{\alpha \in \Sigma_{0}^{+}} x_{k} H_{\alpha_{k}}\right) d x_{1} \cdots d x_{q}=\int_{\mathbf{R}_{+}^{r}} f\left(\sum_{i=1}^{r} x_{i} H_{\alpha_{i}}\right) T\left(\sum_{i=1}^{r} x_{i} H_{\alpha_{i}}\right) d x_{1} \cdots d x_{r} .
$$

Here the $\alpha_{i}$ 's, $i=1, \ldots, r$, are the simple positive roots and $H_{\alpha}$ is defined by the relation $\left(H, H_{\alpha}\right)=\alpha(H)$ for all $H \in \mathfrak{a}$. This led ([14, Prop. 3.1]) to the following explicit formula for $k^{W}$ when $X, Y \in \mathfrak{a}^{+}$:

$$
k^{W}(H, X, Y)=\frac{\pi(\rho)}{|W|} \frac{\sum_{v, w \in W} \epsilon(v) \epsilon(w) T(v X+w Y-H)}{\delta^{1 / 2}(X) \delta^{1 / 2}(Y) \delta^{1 / 2}(H)} .
$$

Expression (3.6) was the main tool of the proof of the support formula (3.1) on $\mathbf{S L}(3, \mathbf{C}) / \mathbf{S U}(3)$.

3.3. Integral formulas for the density and its regularity. In the Introduction, we presented briefly the Fourier-based approach to the convolution of orbital measures. We exploited this approach in [11].

In $\left[\mathbf{1 1}\right.$, Th.3.8], we proved the following integral formula for the density of $\mu_{X, Y}^{W}$. Let $\mathfrak{a}^{\prime}$ denote the set of regular points $W \cdot \mathfrak{a}^{+}$. Then we can write

$$
k^{W}(H, X, Y)=|W|^{-1} \int_{\mathfrak{a}^{*}} \phi_{\lambda}\left(e^{X}\right) \phi_{\lambda}\left(e^{Y}\right) \phi_{\lambda}\left(e^{H}\right)|c(\lambda)|^{-2} d \lambda
$$

on $\mathfrak{a}^{\prime} \times \mathfrak{a}^{\prime} \times \mathfrak{a}^{\prime}$, with a few low rank exceptions: $\mathbf{S L}(n, \mathbf{R}), n \leq 4, \mathbf{S L}(2, \mathbf{C}), \mathbf{S O}(1, q)$, $q \leq 2, \mathbf{S O}(p, q), 2 \leq p \leq q \leq 3, \mathbf{S U}(2,2), \mathbf{S p}(2, \mathbf{R})$ and $\mathbf{S O}^{*}(4)$.

The proof was based on an extension of the inversion formula for the spherical Fourier transform $\mathcal{H}$ :

$$
k^{W}(H, X, Y)=\mathcal{H}^{-1}\left(\phi_{\lambda}\left(e^{X}\right) \phi_{\lambda}\left(e^{Y}\right)\right)
$$


and on the following estimates of the spherical functions from [3]. If $B$ is a compact set of $\mathfrak{a}^{+}$, then there exists a constant $C_{B}$ such that for every $H \in B$ and $\lambda \in\left(\mathfrak{a}^{*}\right)^{+}$

$$
\left|\phi_{\lambda}\left(e^{H}\right)\right| \leq C_{B} \prod_{\alpha \in \Sigma^{+}}(1+\langle\lambda, \alpha\rangle)^{-m_{\alpha} / 2} .
$$

Formula (3.7) allowed us to deduce the following properties of the kernels $k^{W}(H, X, Y)$ and $k(H, X, Y)$ :

- symmetry: $k(H, X, Y)$ is symmetric in all 3 variables on the set $\mathfrak{a}^{\prime} \times \mathfrak{a}^{\prime} \times$ $\mathfrak{a}^{\prime}$.

- continuity: $k(H, X, Y)$ is continuous in all 3 variables on the set $\mathfrak{a}^{\prime} \times$ $\mathfrak{a}^{\prime} \times \mathfrak{a}^{\prime}$.

- smoothness: using the co-area formula, we proved in $[\mathbf{1 1}$, Th. 3.16] that $k(H, X, Y)$ is smooth in the variable $H$ for each $H=a\left(e^{X} k e^{Y}\right) \in \mathfrak{a}^{+}$ such that the differential of the map $k \rightarrow a\left(e^{X} k e^{Y}\right)$ is surjective at $k$. Thus the density $k(H, X, Y)$ is smooth on $\operatorname{int}\left(S_{X, Y} \backslash I\right)$.

We can apply [11, Cor. 3.10] that says that $k$ is of class $\mathcal{C}^{m}$ in its three variables with $m<(\gamma-2) / 2$ where

$$
\gamma=\inf _{i=1, \ldots, r}\left\{\sum_{\left\{\alpha: \eta_{i}(\alpha) \geq 1\right\}} \frac{m_{\alpha}}{l(\alpha)}\right\}
$$

(the $\alpha_{i}$ 's being the simple roots, $\alpha=\sum_{i=1}^{r} \eta_{i}(\alpha) \alpha_{i}$ and $l(\alpha)=\mid\left\{k: \eta_{k}(\alpha) \geq\right.$ $1\} \mid)$.

We end this section by an open question: when is $k$ bounded?

\section{Symmetric spaces of Euclidean type}

The results of this section concerning the density of the convolution of two orbital measures of an infinitesimal flat symmetric space $\mathfrak{p}$ are new.

There has been some recent literature on symmetric spaces of Euclidean type such as the articles $[\mathbf{1}, \mathbf{2}, \mathbf{3 6}]$. We note that the paper $[\mathbf{3 6}]$ by Wolf is a good introduction to the topic of symmetric spaces of Euclidean type or flat symmetric spaces. They are defined by $\mathbf{R}^{n} \simeq G / K$ where $G=K \rtimes \mathbf{R}^{n}$ and $K$ is a subgroup of $\mathbf{O}(n)$. We consider three different situations:

(1) the "maximal" case: $\mathbf{R}^{n} \simeq \mathbf{O}(n) \rtimes \mathbf{R}^{n} / \mathbf{O}(n)$,

(2) the minimal case where $K=\{e\}$.

(3) the Cartan motion group $G_{0}=K \rtimes \mathfrak{p}$ where $\mathfrak{p} \simeq G_{0} / K$ is called an infinitesimal flat symmetric space

4.1. Existence of the density. We discussed the first case in the Introduction since we felt that it was a good "classical" introduction to the questions discussed in this survey. There, we said that the density of the measure $m_{X, Y}$ exists if and only if both $X$ and $Y$ are nonzero. Using the inverse Fourier transform approach, which we used to obtain formula (3.7), and the expression given in (1.1), the density of $m_{X, Y}$ is given in $[\mathbf{2 7}]$ as

$$
K\left(r, r_{1}, r_{2}\right)=\frac{\Gamma(n / 2)}{2^{n-3} \Gamma((n-1) / 2) \sqrt{\pi}} \frac{\left\{\left[r^{2}-\left(r_{1}-r_{2}\right)^{2}\right]\left[\left(r_{1}+r_{2}\right)^{2}-r^{2}\right]\right\}^{(n-3) / 2}}{\left(r r_{1} r_{2}\right)^{n-2}}
$$

on its support which is $\left\{r:\left|r_{1}-r_{2}\right| \leq r \leq r_{1}+r_{2}\right\}$ (here $r_{1}=\|x\|$ and $r_{2}=\|y\|$ ). 
(2) The other extreme case, when $K=\{e\}$, is trivial: in this case, $\delta_{X}^{\natural}=\delta_{X}$ and the support of the measure $m_{X, Y}=\delta_{X} \star \delta_{Y}$ is $\{X+Y\}$. The measure $m_{X, Y}$ is therefore never absolutely continuous in this case.

(3) We will discuss here at some length the more interesting intermediate case, the situation of the Cartan motion group. We start by providing some details and notation.

The group operation in $G_{0}=K \rtimes \mathfrak{p}$ is given by

$$
\left(k_{1}, X\right) \cdot\left(k_{2}, Y\right)=\left(k_{1} k_{2}, X+k_{1} \cdot Y\right)
$$

where we write $k \cdot X$ instead of $\operatorname{Ad}(k) X$. Note that $X \in \mathfrak{p}$ is identified with $(e, X) \in G_{0}$ and $k \in K$ is identified with $(k, 0) \in G_{0}$. by

Recall that the convolution of two probability measures $\mu$ and $\nu$ on $G_{0}$ is defined

$$
(\mu \star \nu)(f)=\int_{G_{0} \times G_{0}} f(X Y) d \mu(X) d \nu(Y)
$$

for a test function on $G_{0}$.

\section{Proposition 4.1.}

(i) The multiplicative convolution of $K$-invariant measures on $G_{0}$ corresponds to the additive convolution of their images on $\mathfrak{p}$.

(ii) Let $X, Y \in \mathfrak{p}$. The support of $\delta_{X}^{\natural} \star \delta_{Y}^{\natural}$ as a measure on $\mathfrak{p}$ is $\operatorname{Ad}(K) X+$ $A d(K) Y$.

Proof. We have

$$
\begin{aligned}
\delta_{X}^{\natural}(f) & =\int_{G_{0}} \int_{K \times K} f\left(\left(k_{1}, 0\right)\left(k_{2}, X_{0}\right)\left(k_{3}, 0\right)\right) d k_{1} d k_{3} d \delta_{X}\left(k_{2}, X_{0}\right) \\
& =\int_{G_{0}} \int_{K \times K} f\left(k_{1} k_{2} k_{3}, k_{1} X_{0}\right) d k_{1} d k_{3} d \delta_{X}\left(k_{2}, X_{0}\right) \\
& =\int_{G_{0}} \int_{K \times K} f\left(k_{1} e k_{3}, k_{1} X\right) d k_{1} d k_{3} \\
& =\int_{K \times K} f\left(k_{1}, k_{2} X\right) d k_{1} d k_{2}
\end{aligned}
$$

and

$$
\delta_{Y}^{\natural}(f)=\int_{K \times K} f\left(k_{1}, k_{2} Y\right) d k_{1} d k_{2} .
$$

Therefore,

$$
\begin{aligned}
\delta_{X}^{\natural} \star \delta_{Y}^{\natural}(f) & =\int_{K \times K \times K \times K} f\left(\left(k_{1}, k_{2} X\right) \cdot\left(k_{3}, k_{4} Y\right)\right) d k_{1} d k_{2} d k_{3} d k_{4} \\
& =\int_{K \times K \times K \times K} f\left(k_{1} k_{3}, k_{1} k_{4} \cdot Y+k_{2} \cdot X\right) d k_{1} d k_{2} d k_{3} d k_{4} \\
& =\int_{K \times K \times K} f\left(k_{3}, k_{4} \cdot Y+k_{2} \cdot X\right) d k_{2} d k_{3} d k_{4} .
\end{aligned}
$$

If $f$ is $K$-invariant then

$$
\delta_{X}^{\natural} \star \delta_{Y}^{\natural}(f)=\int_{K \times K} f\left(e, k_{1} \cdot X+k_{2} \cdot Y\right) d k_{1} d k_{2}
$$


CONVOLUTION OF ORBITAL MEASURES ON SYMMETRIC SPACES: A SURVEY 23

and the support of $\delta_{X}^{\natural} \star \delta_{Y}^{\natural}$ is then $\operatorname{Ad}(K) X+\operatorname{Ad}(K) Y$.

We now consider the Euclidean analogue of Theorem 2.3, part 7:

Proposition 4.2. The support $A d(K) X+A d(K) Y$ of $\delta_{X}^{\natural} \star \delta_{Y}^{\natural}$ has nonempty interior if and only if there exists $k \in K$ such that

$$
V_{X}+\operatorname{Ad}(k) V_{Y}=\mathfrak{p} .
$$

Proof. Let $T: K \times K \rightarrow \mathfrak{p}$ be defined by $T\left(k_{1}, k_{2}\right)=\operatorname{Ad}\left(k_{1}\right) X+\operatorname{Ad}\left(k_{2}\right) Y$. Its derivative at $(A, B) \in \mathfrak{k} \times \mathfrak{k}$ is given by

$$
\begin{aligned}
& d T(A, B)=\left.\frac{d}{d t}\right|_{t=0}\left(\operatorname{Ad}\left(e^{t A} k_{1}\right) X+\operatorname{Ad}\left(e^{B} k_{2}\right) Y\right) \\
& =\left.\frac{d}{d t}\right|_{t=0}\left(\operatorname{Ad}\left(e^{t A}\right) \operatorname{Ad}\left(k_{1}\right) X+\operatorname{Ad}\left(e^{t B}\right) \operatorname{Ad}\left(k_{2}\right) Y\right) \\
& =\left.\frac{d}{d t}\right|_{t=0}\left(e^{t \operatorname{ad}(A)} \operatorname{Ad}\left(k_{1}\right) X+e^{t \operatorname{ad}(B)} \operatorname{Ad}\left(k_{2}\right) Y\right) \\
& =\left[A, \operatorname{Ad}\left(k_{1}\right) X\right]+\left[B, \operatorname{Ad}\left(k_{2}\right) Y\right] \\
& =\operatorname{Ad}\left(k_{1}\right)\left(\left[\operatorname{Ad}\left(k_{1}^{-1}\right) A, X\right]+\operatorname{Ad}\left(k_{1}^{-1} k_{2}\right)\left[\operatorname{Ad}\left(k_{2}\right)^{-1} B, Y\right]\right) \\
& =\operatorname{Ad}\left(k_{1}\right)\left(\left[\operatorname{Ad}\left(k_{1}^{-1}\right) A, X\right]+\operatorname{Ad}\left(k_{1}^{-1} k_{2}\right)\left[\operatorname{Ad}\left(k_{2}\right)^{-1} B, Y\right]\right) .
\end{aligned}
$$

We can conclude that $d T$ is surjective if and only if

$$
[\mathfrak{k}, X]+\operatorname{Ad}(k)[\mathfrak{k}, Y]=\mathfrak{p}
$$

for some $k \in K$. Let $W_{Z}=[\mathfrak{k}, Z]$ where $Z \in \mathfrak{a}$. Then

$$
\begin{aligned}
W_{z} & =\operatorname{span}\left\{\left[X_{\alpha}+\theta\left(X_{\alpha}\right), z\right]\right\}_{\alpha \in \Sigma}=\operatorname{span}\left\{\alpha(z)\left(X_{\alpha}-\theta\left(X_{\alpha}\right)\right)\right\}_{\alpha \in \Sigma} \\
& =\operatorname{span}\left\{X_{\alpha}-\theta\left(X_{\alpha}\right): \alpha(z) \neq 0\right\}=V_{z} .
\end{aligned}
$$

Corollary 4.3. The measure $\delta_{X}^{\natural} \star \delta_{Y}^{\natural}$ is absolutely continuous on $\mathfrak{p}$ if and only if the measure $\delta_{e^{X}}^{\natural} * \delta_{e^{Y}}^{\natural}$ is absolutely continuous on $G$. Therefore, Theorem 2.6 and Theorem 2.7 are also valid for their infinitesimal flat symmetric space counterparts.

\subsection{Properties of the density.}

4.2.1. Formulas for the density. In what follows, we will write $k_{\mathfrak{p}}$ for the density of $\nu_{X, Y}^{W}$ (the Weyl-invariant version of $\nu_{X, Y}$ on $\left.\mathfrak{a}\right)$.

Proposition 4.4. In the complex flat symmetric case, we have

$$
k_{\mathfrak{p}}(H, X, Y)=\frac{\pi(\rho)}{|W|} \frac{\sum_{v, w \in W} \epsilon(v) \epsilon(w) T(v X+w Y-H)}{\prod_{\alpha \in \Sigma_{0}^{+}}[2 \alpha(X)] \prod_{\alpha \in \Sigma_{0}^{+}}[2 \alpha(Y)] \prod_{\alpha \in \Sigma_{0}^{+}}[2 \alpha(H)]}
$$

where $T$ is as in (3.5), and

$$
k_{\mathfrak{p}}(H, X, Y)=\frac{\delta(H)^{1 / 2} \delta(X)^{1 / 2} \delta(Y)^{1 / 2}}{2^{3\left|\Sigma_{0}^{+}\right|} \prod_{\alpha \in \Sigma_{0}^{+}}[\alpha(H) \alpha(X) \alpha(Y)]} k^{W}(H, X, Y),
$$

where $k^{W}$ is the density of the Cartan-transport of the convolution of orbital measures in the curved case, given by(3.6). 
Proof. Recall that the spherical functions on the flat symmetric space $\mathfrak{p}$ are given by the formula([15], [17, Prop. 4.10, Chapter IV])

$$
\Psi_{\lambda}(X)=\frac{\pi(\rho)}{\pi(i \lambda)} \frac{\sum_{w \in W}(\operatorname{det} w) e^{i(w \lambda(X))}}{\prod_{\alpha \in \Sigma_{0}^{+}}[2 \alpha(X)]}
$$

(here $\Sigma_{0}^{+}$is the set of positive roots, $\pi(\lambda)=\prod_{\alpha \in \Sigma_{0}^{+}}\langle\alpha, \lambda\rangle$ and $\rho$ is the half-sum of the positive roots).

It follows that

$$
\Psi_{\lambda}(X)=\frac{\delta(X)^{1 / 2}}{\prod_{\alpha \in \Sigma_{0}^{+}}[2 \alpha(X)]} \phi_{\lambda}\left(e^{X}\right)
$$

Setting

$$
k_{\mathfrak{p}}(H, X, Y)=\frac{\delta(H)^{1 / 2}}{\prod_{\alpha \in \Sigma_{0}^{+}}[2 \alpha(H)]} \frac{\delta(X)^{1 / 2}}{\prod_{\alpha \in \Sigma_{0}^{+}}[2 \alpha(X)]} \frac{\delta(Y)^{1 / 2}}{\prod_{\alpha \in \Sigma_{0}^{+}}[2 \alpha(Y)]} k(H, X, Y)
$$

where $k$ is as in (3.6), we have using (1.3):

$$
\Psi_{\lambda}(X) \Psi_{\lambda}(Y)=\int_{\mathfrak{a}} \Psi_{\lambda}(H) \prod_{\alpha \in \Sigma_{0}^{+}} k_{\mathfrak{p}}(H, X, Y)[2 \alpha(H)]^{2} d H
$$

4.2.2. Thompson's conjecture. The Thompson's conjecture says that for $X$, $Y \in \mathfrak{p}$, the support of $\mu_{X, Y}=\left(\delta_{e^{X}}^{\natural} * \delta_{e^{Y}}^{\natural}\right) \circ a^{-1}$ (where $a$ is the Cartan projection $G \rightarrow \overline{\mathfrak{a}^{+}}$) is equal to the support of $\nu_{X, Y}=\left(\delta_{X}^{\natural} \star \delta_{Y}^{\natural}\right) \circ \tilde{a}^{-1}$ (where $\tilde{a}$ is the Cartan projection $\left.\mathfrak{p}=\operatorname{Ad}(K) \mathfrak{a} \rightarrow \overline{\mathfrak{a}^{+}}\right)$, i.e.

$$
a\left(e^{X} K e^{Y}\right)=\tilde{a}(\operatorname{Ad}(K) X+\operatorname{Ad}(K) Y) .
$$

TheOREM 4.5. The Thompson's conjecture is true when $G / K$ is a Riemannian symmetric space of non-compact type with $G$ complex.

Proof. For regular $X$ and $Y$, this is a direct consequence of formula (4.1). For singular $X$ and $Y$, we approximate them by regular $X$ and $Y$ and we use the continuity of the Cartan projections $a$ and $\tilde{a}$.

The Thompson's conjecture was first proved by Klyachko in [26] for complex simple groups $G$. His proof, based on a lifting of random walks from the group $G$ to its Lie algebra $\mathfrak{g}$ and on the Fourier-based approach (generalization of formula (3.7) to convolutions of $n$ measures) contains gaps (unjustified convergence of (3.7)-like integrals). Our proof of Theorem 4.5 is very short and straightforward.

A proof of the Thompson's conjecture in the general case was given by Kapovich, Leeb, Milson in [25]. It is challenging to prove it by methods of spherical harmonic analysis. Our simple proof of Theorem 4.5 in the complex case suggests that this is feasible.

Remark 4.6. 1. Corollary 4.3 follows evidently from the Thompson conjecture. However the proof using Proposition 4.2 is elementary.

2. Observe that by Thompson's conjecture, the results of Section 3.1 concerning the support of $\mu_{X, Y}$ extend to the support of $\nu_{X, Y}$. 
4.2.3. Rational limits. The results of sections 4.1 and 4.2 are less surprising if we look at the Cartan motion group case as a "rational limit" of the curved counterpart (consider $[\mathbf{1}, \mathbf{2}]$ ). In $[\mathbf{2}]$, the spherical functions $\Psi_{\lambda}(X)$ on $G_{0}$ are shown to be the limits of the spherical functions on the group $G$ :

$$
\Psi_{\lambda}(X)=\lim _{\epsilon \rightarrow 0} \phi_{\lambda / \epsilon}\left(e^{\epsilon X}\right)
$$

Let us show as in [2], as an illustration of this method, how the well-known formula for the spherical functions in the case of symmetric spaces of complex type (see for instance [17, Th. 5.7, Chapter IV]) leads to the Euclidean counterpart (4.2):

$$
\begin{aligned}
\Psi_{\lambda}(X)=\lim _{\epsilon \rightarrow 0} \phi_{\lambda / \epsilon}\left(e^{\epsilon X}\right) & =\lim _{\epsilon \rightarrow 0} \frac{\pi(\rho)}{\pi(i \lambda / \epsilon)} \frac{\sum_{w \in W}(\operatorname{det} w) e^{i(w(\lambda / \epsilon)(\epsilon X))}}{\prod_{\alpha \in \Sigma_{0}^{+}}[2 \sinh \alpha(\epsilon H)]} \\
& =\lim _{\epsilon \rightarrow 0} \frac{\pi(\rho)}{\pi(i \lambda)} \frac{\sum_{w \in W}(\operatorname{det} w) e^{i(w \lambda(X))}}{\prod_{\alpha \in \Sigma_{0}^{+}}[2 \sinh \alpha(\epsilon H) / \epsilon]} \\
& =\frac{\pi(\rho)}{\pi(i \lambda)} \frac{\sum_{w \in W}(\operatorname{det} w) e^{i(w \lambda(X))}}{\prod_{\alpha \in \Sigma_{0}^{+}}[2 \alpha(X)]} .
\end{aligned}
$$

If we proceed naively, i.e. without justifying taking the limit inside the integral, we have in the general case

$$
\begin{aligned}
& \Psi_{\lambda}(X) \Psi_{\lambda}(Y) \\
& =\lim _{\epsilon \rightarrow 0} \phi_{\lambda / \epsilon}\left(e^{\epsilon X}\right) \phi_{\lambda / \epsilon}\left(e^{\epsilon Y}\right) \\
& =\lim _{\epsilon \rightarrow 0} \int_{\mathfrak{a}} \phi_{\lambda / \epsilon}\left(e^{h}\right) k(H, \epsilon X, \epsilon Y) \delta(h) d H \\
& =\lim _{\epsilon \rightarrow 0} \epsilon^{r} \int_{\mathfrak{a}} \phi_{\lambda / \epsilon}\left(e^{\epsilon H}\right) k(\epsilon H, \epsilon X, \epsilon Y) \delta(\epsilon H) d H \\
& =\lim _{\epsilon \rightarrow 0} \int_{\mathfrak{a}} \phi_{\lambda / \epsilon}\left(e^{\epsilon H}\right) \epsilon^{r+N} k(\epsilon H, \epsilon X, \epsilon Y) \frac{\delta(\epsilon H)}{\epsilon^{N}} d H \\
& =\int_{\mathfrak{a}} \Psi_{\lambda}(H)\left(\lim _{\epsilon \rightarrow 0} \epsilon^{r+N} k(\epsilon H, \epsilon X, \epsilon Y)\right) \prod_{\alpha \in \Sigma_{0}^{+}}[2 \alpha(H)]^{m_{\alpha}} d H
\end{aligned}
$$

where $r$ is the rank and $N=\Sigma_{\alpha>0} m_{\alpha}$. We conjecture therefore the following limit expression:

$$
k_{\mathfrak{p}}(H, X, Y)=\lim _{\epsilon \rightarrow 0} \epsilon^{r+N} k(\epsilon H, \epsilon X, \epsilon Y) .
$$

To see that (4.4) is true in the complex case, one only has to observe that $\lim _{\epsilon \rightarrow 0} \epsilon^{-N} \delta(\epsilon X)=\prod_{\alpha \in \Sigma_{0}^{+}}[2 \alpha(X)]^{2}$ and that $T(\epsilon H)=\epsilon^{N / 2-r} T(H)$ (clear from the definition of $T$ and the fact that $\left.q=\left|\Sigma_{0}^{+}\right|=N / 2\right)$. 
It is interesting to note, still using the same naive approach and using the expression (3.7), we have

$$
\begin{aligned}
k_{\mathfrak{p}}(H, X, Y) & =\lim _{\epsilon \rightarrow 0} \epsilon^{r+N} k(\epsilon H, \epsilon X, \epsilon Y) \\
& =\lim _{\epsilon \rightarrow 0}|W|^{-1} \epsilon^{r+N} \int_{\mathfrak{a}^{*}} \phi_{\lambda}\left(e^{\epsilon X}\right) \phi_{\lambda}\left(e^{\epsilon Y}\right) \phi_{\lambda}\left(e^{\epsilon H}\right)|c(\lambda)|^{-2} d \lambda \\
& =\lim _{\epsilon \rightarrow 0}|W|^{-1} \int_{\mathfrak{a}^{*}} \phi_{\lambda / \epsilon}\left(e^{\epsilon X}\right) \phi_{\lambda / \epsilon}\left(e^{\epsilon Y}\right) \phi_{\lambda / \epsilon}\left(e^{\epsilon H}\right)\left(\epsilon^{-N}|c(\lambda / \epsilon)|^{-2}\right) d \lambda \\
& =C_{0} \int_{\mathfrak{a}^{*}} \Psi_{\lambda}(X) \Psi_{\lambda}(Y) \Psi_{\lambda}(H) \prod_{\alpha \in \Sigma_{0}^{+}}\left\langle\lambda, \alpha_{0}\right\rangle^{m_{\alpha}+m_{2 \alpha}} d \lambda
\end{aligned}
$$

$\left(\alpha_{0}=\alpha /\langle\alpha, \alpha\rangle\right)$ noting that

$$
\lim _{\epsilon \rightarrow 0} \epsilon^{N}|c(\lambda / \epsilon)|^{-2}=c_{0}^{-2}(4 \pi)^{2\left|\Sigma_{0}^{+}\right|} 2^{-N+\left|\Sigma_{0}^{+}\right|} e^{-N-\left|\Sigma_{0}^{+}\right|} \prod_{\alpha \in \Sigma_{0}^{+}}\left\langle\lambda, \alpha_{0}\right\rangle^{m_{\alpha}+m_{2 \alpha}} .
$$

We used the fact that $|c(\lambda)|^{2}=c(\lambda) c(-\lambda)$, that $\Gamma(a+b i) \Gamma(a-b i)=|\Gamma(a+b i)|^{2}$ when $a$ and $b$ are real, $|\Gamma(a+b i)|=\sqrt{2 \pi}|b|^{a-1 / 2} e^{-a-|b| \pi / 2}[1+O(1 /|b|)]$ when $|b| \rightarrow \infty($ refer to $[\mathbf{2 2}])$ and

$$
c(\lambda)=c_{0} \prod_{\alpha \in \Sigma_{0}^{+}} \frac{2^{-i\left\langle\lambda, \alpha_{0}\right\rangle} \Gamma\left(i\left\langle\lambda, \alpha_{0}\right\rangle\right)}{\Gamma\left(\frac { 1 } { 2 } ( \frac { 1 } { 2 } m _ { \alpha } + 1 + i \langle \lambda , \alpha _ { 0 } \rangle ) \Gamma \left(\frac{1}{2}\left(\frac{1}{2} m_{\alpha}+m_{2 \alpha}+i\left\langle\lambda, \alpha_{0}\right\rangle\right)\right.\right.}
$$

(refer for example to $[\mathbf{1 7}]$ ).

This leads us to a second conjecture: that

$$
k_{\mathfrak{p}}(H, X, Y)=C_{0} \int_{\mathfrak{a}^{*}} \Psi_{\lambda}(X) \Psi_{\lambda}(Y) \Psi_{\lambda}(H) \prod_{\alpha \in \Sigma_{0}^{+}}\left\langle\lambda, \alpha_{0}\right\rangle^{m_{\alpha}+m_{2 \alpha}} d \lambda
$$

holds with the same exceptions as for formula (3.7).

The term $C_{0}\left\langle\lambda, \alpha_{0}\right\rangle^{m_{\alpha}+m_{2 \alpha}} d \lambda$ corresponds to Plancherel measure in that setup (see for instance $[\mathbf{1 7}$, Th. 9.1, Chapter IV] or [28]). One could prove (4.5) formally by using the same approach as in $[\mathbf{1 1}]$ provided that a result similar to the bound given in (3.8) can be found.

\section{References}

[1] J.-P. Anker, F. Ayadi and M. Sifi, Opdam's hypergeometric functions: product formula and convolution structure in dimension 1, Adv. Pure Appl. Math. 3, 11-44, 2012.

[2] S. Ben Saïd, B. Orsted, Analysis on flat symmetric spaces, J. Math. Pures Appl. 84, 13931426, 2005.

[3] J. J. Duistermaat, J. A. Kolk and V. S. Varadajan. Functions, flows and oscillatory integrals on flag manifolds and conjugacy classes in real semisimple Lie groups, Compositio Mathematica 49, 309-398, 1983.

[4] M. Flensted-Jensen, T. Koornwinder, The convolution structure for Jacobi function expansions, Ark. Mat. 11, 245-262, 1973.

[5] P. Graczyk and J.J. Loeb, Spherical analysis and central limit theorems on symmetric spaces, Probability measures on groups and related structures, XI (Oberwolfach, 1994), 146-166, World Sci. Publishing, River Edge, NJ, 1995.

[6] P. Graczyk, J.J. Loeb, T. Zak, Strong central limit theorem for isotropic random walks in $\mathbb{R}^{d}$, Probab. Theory Relat. Fields 151, No. 1-2, 153-172, 2011.

[7] P. Graczyk, P. Sawyer, Convolution of orbital measures on symmetric spaces of type $C_{p}$ and $D_{p}, 1-25$, to appear in J. Australian Math. Soc., 2014. 
[8] P. Graczyk, P. Sawyer, On the product formula on noncompact Grassmannians, Coll.Math. 133, 145-167, 2013.

[9] P. Graczyk, P. Sawyer, A sharp criterion for the existence of the product formula on symmetric spaces of type $A_{n}$, J. Lie Theory 20, 751-766, 2010.

[10] P. Graczyk, P. Sawyer, Absolute continuity of convolutions of orbital measures on Riemannian symmetric spaces, Journal of Functional Analysis 259, 1759-1770, 2010.

[11] P. Graczyk and P. Sawyer, On the kernel of the product formula on symmetric spaces, Journal of Geometric Analysis, Vol. 14, 4, 653-672, 2004.

[12] P. Graczyk and P. Sawyer, Some convexity results for the Cartan decomposition, Canadian Journal of Mathematics, no. 5, 1000-1018, 2003.

[13] P. Graczyk and P. Sawyer, The product formula for the spherical functions on symmetric spaces of noncompact type, Journal of Lie Theory, vol. 13, no. 1, 247-261, 2003.

[14] P. Graczyk and P. Sawyer, The product formula for the spherical functions on symmetric spaces in the complex case, Pacific Journal of Mathematics, vol. 204, no. 2, 377-393, 2002.

[15] Harish-Chandra, Representations of semisimple Lie groups, III, Trans. Amer. Math. Soc. 76, 234-253, 1954.

[16] S. Helgason, Differential Geometry, Lie Groups and Symmetric spaces, Academic Press, New York, 1978.

[17] S. Helgason, Groups and geometric analysis, Academic Press, 1984.

[18] S. Helgason, Geometric analysis on symmetric spaces, Mathematical surveys and monographs, Vol. 39, American Mathematical Society, 1994

[19] B. D. Hughes, Random walks in random environments, Vol. 1, Oxford University Press, 1995.

[20] W. Jaworski, Strong approximate transitivity, polynomial growth, and spread out random walks on locally compact groups. Pacific J. Math. 170, no. 2, 517-533, 1995.

[21] W. Jaworski, C.R.E. Raja,The Choquet-Deny theorem and distal properties of totally disconnected locally compact groups of polynomial growth, New York J. Math. 13, 159-174, 2007.

[22] Kilbas, Anatoly A. and Saigo, Megumi, A Remark on Asymptotics of the Gamma Function at Infinity, (Study on Applications for Fractional Calculus Operators in Univalent Function Theory). RIMS Kokyuroku, 1363, 33-36, 2004.

[23] T. Koornwinder, Jacobi polynomials. II. An analytic proof of the product formula, SIAM J. Math. Anal. 5, 125-137, 1974.

[24] M. Lin and R. Wittmann, Convolution powers of spread-out probabilities, Ann. Inst. H. Poincaré Probab. Statist. 32, no. 5, 661-667, 1996.

[25] M. Kapovich, Michael, B. Leeb, J. J. Millson, Polygons in buildings and their refined side lengths, Geom. Funct. Anal. 19, no. 4, 1081-1100, 2009.

[26] A. Klyachko, Random walks on symmetric spaces and inequalities for matrix spectra, Special Issue: Workshop on Geometric and Combinatorial Methods in the Hermitian Sum Spectral Problem (Coimbra, 1999), Linear Algebra Appl. 319, no. 1-3, 3-59, 2000.

[27] I.V. Ostrovskii, Description of the $I_{0}$ class in a special semigroup of probability measures, Sov. Math. Dokl. 14, 525-529 (1973); translation from Dokl. Akad. Nauk SSSR 209, 788-791, 1973.

[28] C. Rader, Spherical Functions on Cartan Motion Groups, Transactions of the American Mathematical Society, Vol. 310, No. 1, 1-45, 1988.

[29] D. L. Ragozin, Zonal measure algebras on isotropy irreducible homogeneous spaces, J. Functional Analysis, vol. 17, 355-376, 1974.

[30] M. Rösler, Positive convolution structure for a class of Heckman-Opdam hypergeometric functions of type BC, J. Funct. Anal. 258, no. 8, 2779-2800, 2010.

[31] P. Sawyer, The product formula on the space $\mathbf{S O}^{*}(2 n) / \mathbf{U}(n), 1-11,2014$.

[32] P. Sawyer, Spherical functions on $\mathbf{S O}_{0}(p, q) / \mathbf{S O}(p) \times \mathbf{S O}(q)$, Canadian Mathematical Bulletin, vol. 42, no. 4, 1999, 486-498, 1999 .

[33] R. C. Thompson, Matrix spectral inequalities, Johns Hopkins Univ. Press, Baltimore, MD, 1988.

[34] I.P. Trukhina, The arithmetic of spherically symmetric measures in Lobachevskij space, Teor. Funkts. Funkts. Anal. Prilozh. 34, 136-146, 1980.

[35] M. Voit, Factorization of probability measures on symmetric hypergroups, J. Aust. Math. Soc., Ser. A 50, No.3, 417-467, 1991. 
[36] J. A. Wolf, Spherical functions on Euclidean space, Journal of Functional Analysis, 239, 127-136, 2006.

Laboratoire de Mathématiques, LAREMA, Université d’Angers, 49045 Angers Cedex

01

E-mail address: piotr.graczyk@univ-angers.fr

Department of Mathematics, and Computer Science, Laurentian University, SudBURY, ONTARIO

E-mail address: psawyer@laurentian.ca 